ARTICLE

\title{
Heteromeric HSFA2/HSFA3 complexes drive transcriptional memory after heat stress in Arabidopsis
}

Thomas Friedrich (10 1, Vicky Oberkofler ${ }^{1}$, Inês Trindade ${ }^{1}$, Simone Altmann ${ }^{1,3}$, Krzysztof Brzezinka ${ }^{1}$, Jörn Lämke , Michal Gorka², Christian Kappel (1) 1, Ewelina Sokolowska², Aleksandra Skirycz (1) ${ }^{2}$, Alexander Graf ${ }^{2}$ \& Isabel Bäurle (iD) ${ }^{1 凶}$

Adaptive plasticity in stress responses is a key element of plant survival strategies. For instance, moderate heat stress (HS) primes a plant to acquire thermotolerance, which allows subsequent survival of more severe HS conditions. Acquired thermotolerance is actively maintained over several days (HS memory) and involves the sustained induction of memoryrelated genes. Here we show that FORGETTER3/ HEAT SHOCK TRANSCRIPTION FACTOR A3 (FGT3/HSFA3) is specifically required for physiological HS memory and maintaining high memory-gene expression during the days following a HS exposure. HSFA3 mediates HS memory by direct transcriptional activation of memory-related genes after return to normal growth temperatures. HSFA3 binds HSFA2, and in vivo both proteins form heteromeric complexes with additional HSFs. Our results indicate that only complexes containing both HSFA2 and HSFA3 efficiently promote transcriptional memory by positively influencing histone $\mathrm{H} 3$ lysine 4 (H3K4) hyper-methylation. In summary, our work defines the major HSF complex controlling transcriptional memory and elucidates the in vivo dynamics of HSF complexes during somatic stress memory.

\footnotetext{
${ }^{1}$ Institute for Biochemistry and Biology, University of Potsdam, Potsdam, Germany. ${ }^{2}$ Max-Planck-Institute for Molecular Plant Physiology, Potsdam, Germany.

${ }^{3}$ Present address: School of Life Sciences, University of Dundee, Dundee, UK. ${ }^{\circledR}$ email: isabel.baeurle@uni-potsdam.de
} 
M any organisms are frequently exposed to adverse environmental conditions that interfere with their development and growth and are referred to as stress. Plants can acclimate to stress conditions, and a transient stress cue can prime plants for a modified defense response upon exposure to a recurring stress after a stress-less interval ${ }^{1-3}$. This so-called somatic stress memory has been described in response to a number of different biotic and abiotic stress cues (reviewed in refs. ${ }^{3-5}$ ). Occasionally, stress-memory may also extend into future generations ${ }^{6-8}$. Somatic transcriptional memory based on enhanced re-induction of stress-induced genes following a second stress exposure has been reported for drought stress $^{9,10}$, salt stress $^{11}$ and for defense-related priming ${ }^{12-14}$. In these cases chromatin modifications, in particular histone $\mathrm{H} 3$ lysine 4 (H3K4) methylation have been correlated with transcriptional memory $9,11-13$. However, the mechanistic basis of stress-induced transcriptional memory and its conservation across different phenomena remains poorly understood.

A major factor limiting global crop yields is heat stress (HS), and it is predicted that its prevalence will increase with climate change ${ }^{15,16}$. In response to acute HS, plants acquire thermotolerance and this is molecularly very similar to the HS response (HSR) of yeast and metazoans ${ }^{17-20}$. However, in nature, HS is often recurring, and plants can be primed by one HS for an improved response to a recurring HS after a stress-less lag phase of several days $3,21,22$. This HS memory is an active process as it is genetically separable from the acquisition of thermotolerance, and several genes have been identified that function specifically in HS memory ${ }^{21-25}$.

An essential component of transcriptional HS responses across kingdoms is their activation through HEAT SHOCK FACTOR (HSF) transcription factors. Interestingly, the activity of HSF proteins is also highly relevant for aging and tumorigenesis ${ }^{18,19}$. While yeast only has one HSF and vertebrates have up to 4, this gene family has radiated massively in higher plants ${ }^{26}$. Of the 21 HSF genes in Arabidopsis thaliana, seven have been implicated in the HSR, among them three isoforms of HSFA1; $A 1 A, A 1 B$, and $A 1 D^{26-31}$. The HSFA1 genes are considered as master regulators that function in a largely analogous manner to yeast and metazoan $\mathrm{HSF}^{20}$. HSFA1 isoforms are constitutively expressed and are posttranslationally activated upon HS. They induce a suite of target genes, including many heat shock proteins (HSPs) that act as chaperones. HSFA2 specifically functions in HS memory ${ }^{22}$ and it is very strongly induced after HS by HSFA1 proteins ${ }^{28,29}$. HSFA2 in turn amplifies the transcriptional induction of a subset of HS-response genes, but is not required for their initial activation. This subset overlaps with the genes that have been classified as HS memory-related genes due to their sustained induction after HS, lasting for at least two days ${ }^{23}$. Interestingly, HSFA2 binds only transiently to these HS memoryrelated genes, while HSFA2-dependent differences in transcriptional activity are mostly observed after binding of HSFA2 has decreased ${ }^{32}$. Chromatin profiling of HS memory-related genes revealed that HSFA2 recruits $\mathrm{H} 3 \mathrm{~K} 4$ hyper-methylation at these loci, and this correlates with the duration of the memory phase (at least $5 \mathrm{~d})^{32,33}$. This HS-induced enrichment likely extends the phase of active transcription at these genes and was not present in highly HS-inducible "non-memory" genes such as HSP70 and HSP101. Thus, the current model is that HSFA2 sustains transcriptional activation through the memory phase by recruiting sustained H3K4 methylation. This mediates a transcriptional memory (type I) that sustains transcriptional activity of certain genes for several days after the end of a short HS. After transcription has subsided, a second type of transcriptional memory remains active at a subset of genes. This causes enhanced transcriptional re-induction upon a recurring HS (type II transcriptional memory) and is active for $6 \mathrm{~d}$ after the priming $\mathrm{HS}^{33}$. HSFA2 is required for both types of transcriptional memory after $\mathrm{HS}^{32}$. However, the mechanistic basis of how HSFA2 promotes HS memory remains poorly understood. It is well established that HSF proteins form trimeric and hexameric complexes in yeast, metazoans and plants ${ }^{18,34,35}$. Yet, major unresolved questions are (1) whether HSFA2 is the only HSF protein in A. thaliana that mediates HS memory, and (2) what the composition of the HSFA2-containing HSF complexes is. (3) More generally, the composition of HSF complexes in vivo at endogenous expression levels is virtually unknown, as is the function of many of the different HSF family members in $A$. thaliana.

To identify additional components required for regulation of HS memory, we have employed a reporter-based genetic screen where the HS memory gene HSA32 was translationally fused to the LUCIFERASE reporter gene ${ }^{24}$. HSA32 shows sustained induction after HS and the corresponding mutant is specifically defective in HS memory at the whole plant level ${ }^{21,24}$. Screening for mutants with normal activation but reduced maintenance of HSA32-LUC expression, we identified the forgetter1 (fgt1) mutant $^{24}$. FGT1 encodes the A. thaliana orthologue of Drosophila strawberry notch, a highly conserved helicase protein that is required to maintain an open chromatin conformation through cooperation with chromatin remodeling complexes of the SWI/ SNF family 24,36 .

Here, we describe the isolation and characterization of the FGT3 gene from the above screen. We show that FGT3 encodes the HSFA3 gene and that the fgt 3 mutant has a HS memoryspecific phenotype, comparable to hsfa2. We provide evidence that HSFA3 is a second key HSF underlying HS memory and that it forms heteromeric complexes with HSFA2 that efficiently promote transcriptional memory. These findings serve not only to assign function to a further important HSF family member, but also provide information about the in vivo composition of HSF complexes in A. thaliana.

\section{Results}

FORGETTER3 (FGT3) is required for HS memory and sustained induction of $\boldsymbol{H S A 3 2}$. To identify factors that are implicated in HS memory, we performed a mutagenesis screen for modifiers of HS-induced sustained expression of pHSA32:: HSA32-LUC ${ }^{24}$. In fgt3 mutants HSA32-LUC induction was normal at $1 \mathrm{~d}$ after a two-step HS treatment ("acclimation", ACC, Fig. 1a), but declined prematurely during the following two days at normal growth temperature (Fig. 1b). In line with this finding fgt 3 mutants also had a defective HS memory at the physiological level (Fig. 1c, Supplementary Fig. 1a). However, the immediate HS responses - as assayed by basal thermotolerance and acquired thermotolerance - were not affected in fgt3 (Supplementary Fig. 1b-c). Thus, FGT3 is specifically required for HS memory.

FGT3 encodes HSFA3. The fgt3 mutant segregated as a single recessive locus with no apparent morphological defects under normal growth conditions. To identify the genetic mutation underlying the HS memory phenotype, we combined recombination breakpoint mapping and genome re-sequencing, and identified a single nucleotide polymorphism in exon 2 of At5g03720 that introduces a premature stop codon in the HSFA3 gene (Fig. 1d). To confirm that fgt3 is allelic to $h s f a 3$, we crossed fgt 3 to hsfa3-1 and assayed HSA32-LUC activity in the F1 progeny after HS. The progeny of the fgt $3 \times$ x $h f a 3-1$ cross, but not the control cross, showed strongly reduced maintenance of HSA32LUC activity and loss of physiological HS memory, similar to fgt 3 (Fig. 1b, Supplementary Fig. 2a). In addition, genomic constructs 


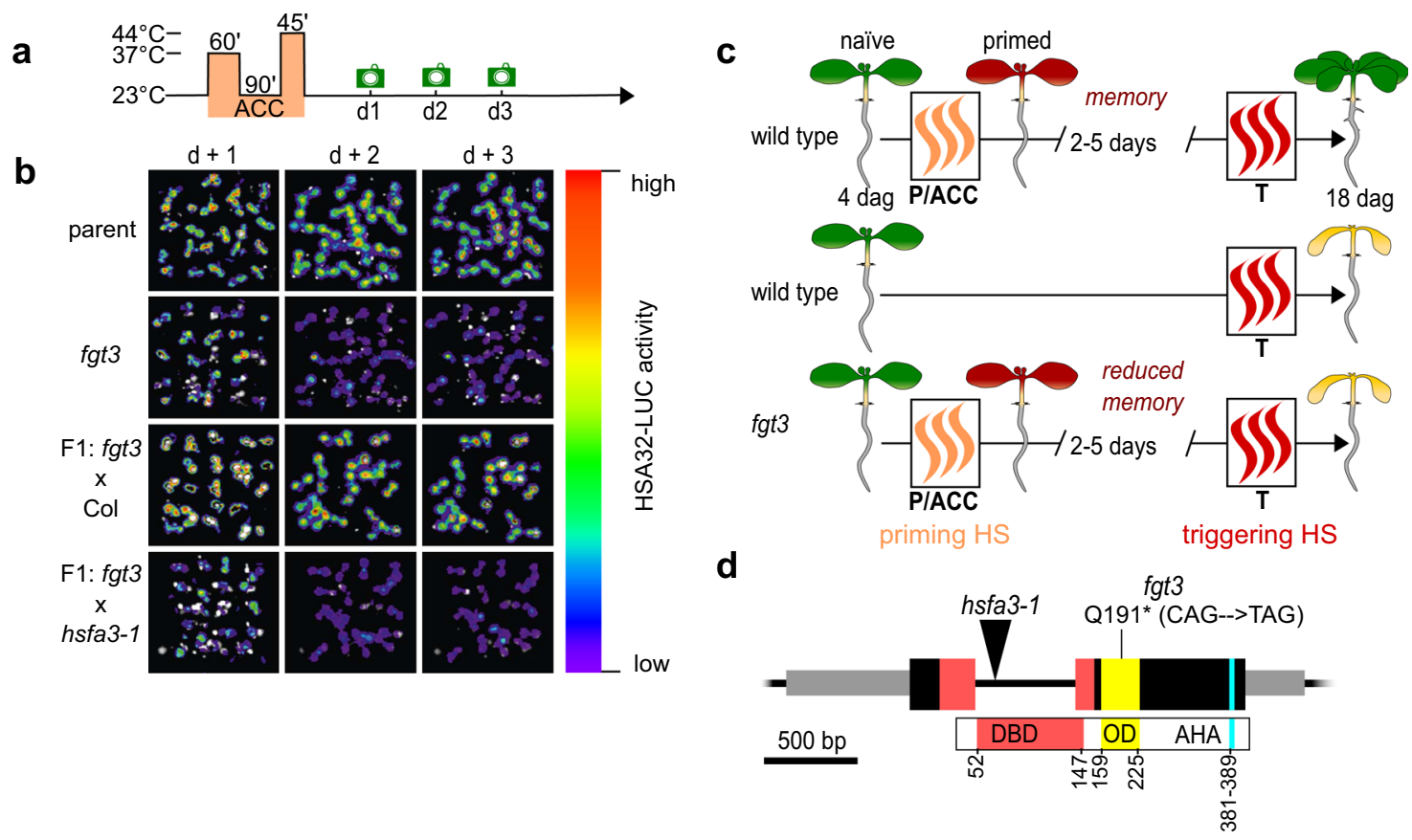

Fig. 1 FGT3/HSFA3 is required for HS memory. a Treatment scheme for LUC-based HS memory assay: 4 d-old seedlings were exposed to a two-step acclimation treatment (ACC; $60 \mathrm{~min} 37^{\circ} \mathrm{C}, 90 \mathrm{~min} 23^{\circ} \mathrm{C}$ and $45 \mathrm{~min} 44^{\circ} \mathrm{C}$ ). Activity of the HS memory marker pHSA32::HSA32-LUC was scored on the following three days (green camera symbols). b LUC-based HS memory assay shows reduced maintenance of pHSA32::HSA32-LUC induction in forgetter 3 (fgt3) mutants. Crossing to Col but not to the hsfa3-1 mutant complements the defect of fgt3 in the F1 progeny. c Schematic representation of physiological HS memory: Plants that have not experienced any HS (naïve plants) can be primed by a non-lethal HS ( $P$ or ACC), leading to an enhanced capacity to withstand a triggering HS (T). This enhanced thermotolerance results in increased survival of $\mathrm{T}$ in a primed plant compared to a naïve plant for up to $5 \mathrm{~d}$ (HS memory). Fgt3 mutants are defective in HS memory and do not survive the T despite prior priming. d Schematic representation of the HSFA3 locus (At5g03720) with location of the fgt3 (Q191*) and hsfa3-1 mutations. Exons are shown as large black boxes with protein domains overlaid in color (DBD: DNA-binding domain, OD: oligomerization domain, AHA: AHA motif), aa numbers are given to depict the positions of protein domains. UTRs are shown as gray boxes and the intron as a black line.

expressing HSFA3 under the control of the endogenous promoter (1.3 kb promoter fragment) with or without an N-terminal FLAG tag complemented HSA32-LUC expression and survival phenotypes of fgt3 (Supplementary Figs. 1a, 2b). The hsfa3-1 allele displayed similar HS memory defects as $f g t 3$ and was also complemented by pHSFA3::FLAG-HSFA3 (Fig. $2 \mathrm{a}-\mathrm{d}$ ). Thus, the loss of $H S F A 3$ function is causative for the fgt 3 mutant phenotypes and we renamed fgt 3 as $h s f a 3-3$.

Under our conditions, HSFA3 induction peaked at $4 \mathrm{~h}$ after the end of ACC (Fig. 2e). We also tested the expression pattern of the two $p H S F A 3:: F L A G-H S F A 3$ lines. Line \#1 expressed FLAGHSFA3 similarly as HSFA3 in Col. Line \#12, however, showed a several-fold stronger induction of FLAG-HSFA3 after ACC, suggesting that this line acts as a native overexpressor (Fig. 2e). Interestingly, line \#12 displayed enhanced HS memory compared to Col (Fig. 2b). Further extending the recovery phase between the priming and triggering HS for up to $6 \mathrm{~d}$ and reducing the dose (duration) of the triggering HS revealed that HS memory in wild type was still detected after $5 \mathrm{~d}$ of recovery, albeit at decreasing levels (Supplementary Fig. 3a, b). This observation extends the memory period regarding physiological effects by $2 \mathrm{~d}$ compared to previous reports ${ }^{23,24}$. Notably, the native overexpressing line retained some HS memory $6 \mathrm{~d}$ after ACC, when primed Col plants no longer had enhanced survival compared to unprimed Col plants. Thus, HSFA3 protein levels control HS memory.

HSFA3 and HSFA2 interact genetically and have redundant and non-redundant functions. HSFA 3 was induced by ACC, albeit more slowly than $H S F A 2$, which peaked right at the end of the ACC treatment (Fig. 3a, b). HSFA3 was suggested to be induced by the HS-activated DEHYDRATION-RESPONSIVE ELEMENT BINDING PROTEIN 2 A (DREB2A), which in turn is activated by HSFA1 isoforms ${ }^{37-39}$. Indeed, under our HS regime HSFA3 but not HSFA2 expression depended on DREB2A, consistent with the predicted presence of more DREB binding elements in the HSFA3 promoter than in the HSFA2 promoter (Supplementary Fig. 3c, Supplementary Table 1). This two-step activation may account for the slower induction kinetics of HSFA3. Consistent with both genes being downstream of HSFA1s, induction of either HSFA2 or HSFA3 was independent of the respective other protein (Fig. $3 \mathrm{a}, \mathrm{b}$ ).

HSFA 2 and HSFA3 are both required for HS memory, with hsfa2 having a slightly stronger defect (Fig. 3c, d). To test whether both genes interact genetically, we generated the hsfa2 hsfa3-1 double mutant and analyzed physiological HS memory. The double mutant was more sensitive to a triggering HS that was applied $3 \mathrm{~d}$ after ACC than either single mutant (Fig. 3c, d). None of the mutants or native overexpressing lines showed any defects in basal or acquired thermotolerance, indicating that the observed phenotypes are specific to HS memory (Supplementary Fig. 4). In summary, despite the already strong phenotypes of the single mutants, double mutant analysis indicates that HSFA2 and HSFA3 act partially redundantly.

HSFA3 is required for sustained induction of HS memoryrelated genes. Two types of HS-related transcriptional memory have been described; type I memory (sustained induction) and type II memory (enhanced re-induction) ${ }^{5,32}$. We asked whether HSFA3 is required for type I memory (sustained induction) by analyzing transcript levels of the endogenous HSA32 gene as well 


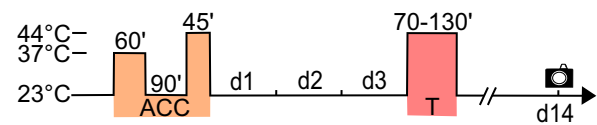

C
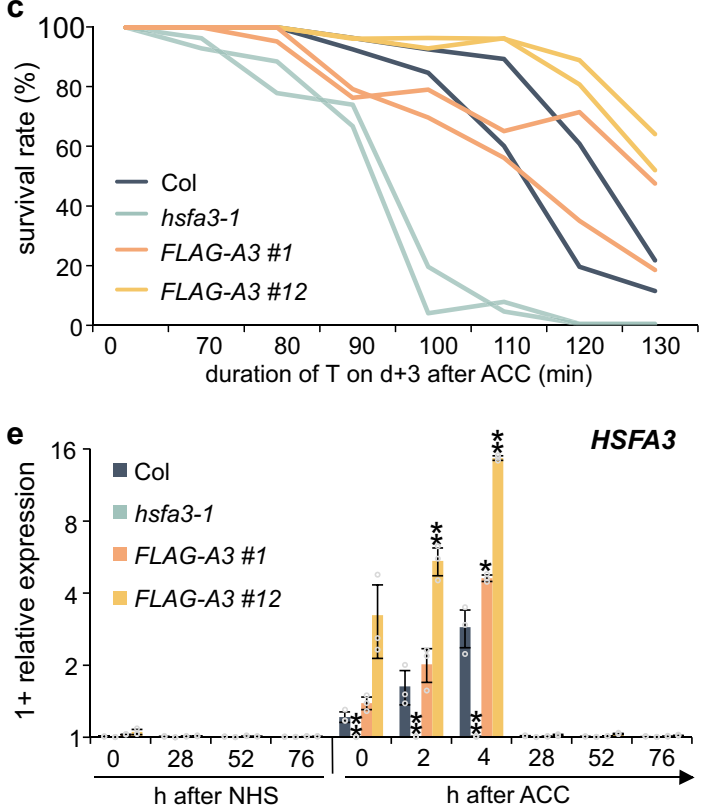

b

b

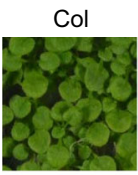

$\mathrm{ACC}+$

$\mathrm{T} \mathrm{d}+3$
$70 \mathrm{~min}$

$70 \mathrm{~min}$ Cis?

$\mathrm{ACC}+$

$90 \mathrm{~min}$

$\mathrm{ACC}+$

$\mathrm{T} d+3$
$110 \mathrm{~min}$
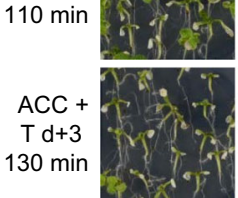

$T d+3$
$130 \mathrm{~min}$
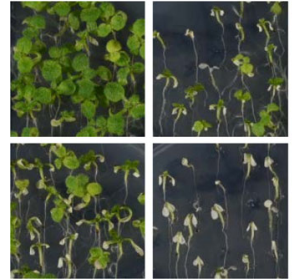

hsfa3-1
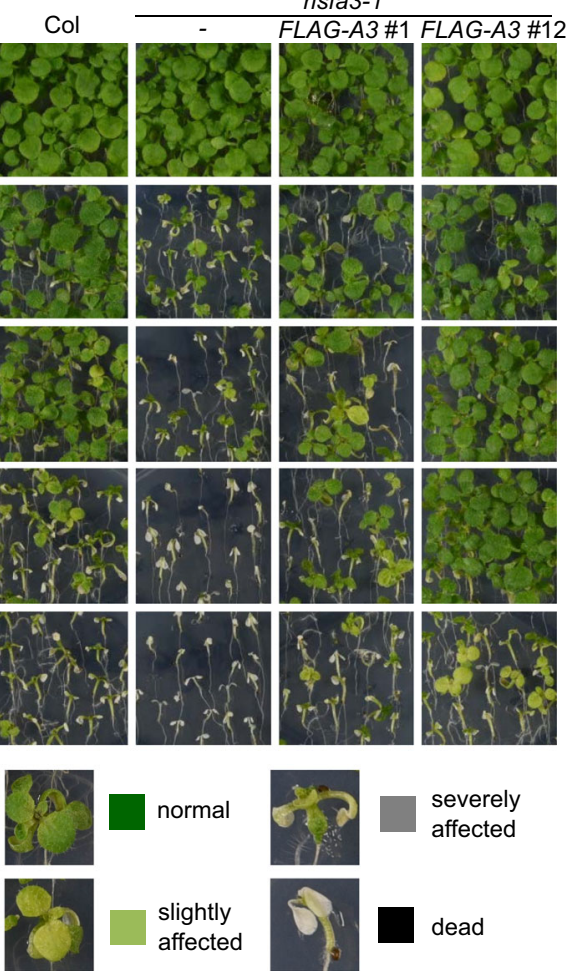

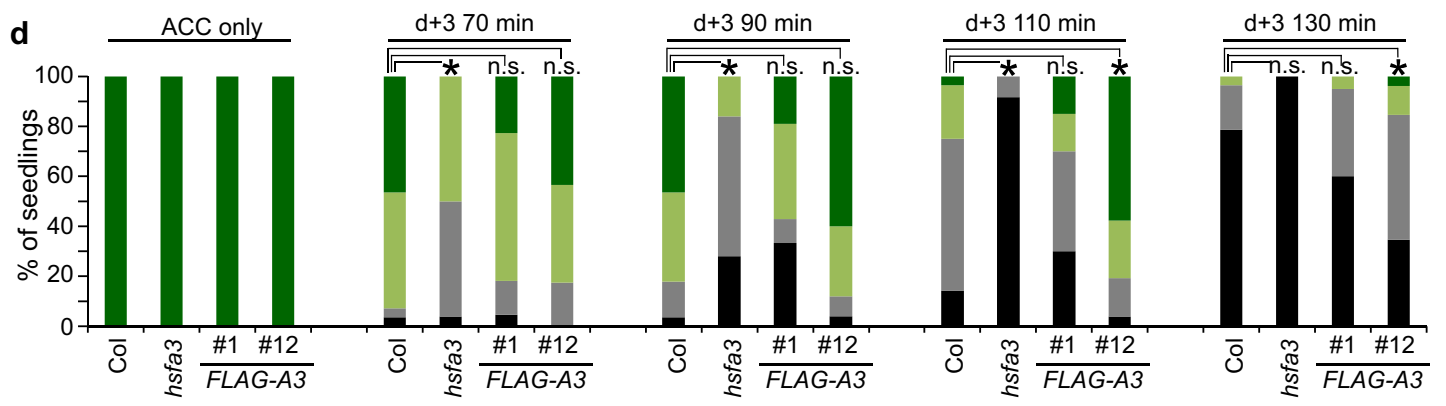

Fig. 2 FGT3/HSFA3 is required for physiological HS memory. a Treatment scheme for $\mathrm{HS}$ memory assays: plants are exposed to a triggering $\mathrm{HS}$ ( $\mathrm{T}$, $44^{\circ} \mathrm{C}$ for 70-130 min) $3 \mathrm{~d}$ after ACC (which was applied $4 \mathrm{~d}$ after germination) and survival is scored $14 \mathrm{~d}$ after ACC (black camera symbol). b-d HS memory assay of hsfa3-1 and two complementing lines of pHSFA3::FLAG-HSFA3 in the hsfa3-1 background. b Representative images of HS memory assay, with legend showing examples of phenotype categories used for the quantification in d. c Survival rates of the different genotypes in HS memory assay. Data are from 2 independent replicates with $n \geq 19$ seedlings for each timepoint and genotype. d Distribution of phenotypic categories observed in the HS memory assay shown in $\mathbf{b}$. Asterisks depict significant differences to Col ( $p<0.01$, Fisher's exact test, $n \geq 19$ seedlings for each timepoint and genotype). e Transcript levels of HSFA3 in Col, hsfa3-1 and two pHSFA3::FLAG-HSFA3 lines in the hsfa3-1 background as measured by qRT-PCR. Expression values are relative to the At4g26410 reference gene, as in all following qRT-PCR figures. Data are mean \pm SD of three independent experiments and asterisks indicate significant differences to $\mathrm{Col}\left({ }^{\star}, p<0.05 ;{ }^{\star \star}, p<0.01\right.$; unpaired two-sided t-test).

as three other genes of this group, HSP18.2, HSP22, and $H S P 21^{23,24,32}$. Starting from $28 \mathrm{~h}$ after ACC, transcript levels of these genes in $h s f a 3-1$ were lower compared to wild type, indicating a defect in sustained induction, but not initial upregulation (Fig. 4). In contrast, the native overexpressing line \#12 showed increased expression levels of HSA32, HSP22, and APX2 from 28 $\mathrm{h}$ onwards (Supplementary Fig. 5a), in line with the stronger HSFA3 expression after ACC and the enhanced HS memory (Fig. 2). In the hsfa2 hsfa3-1 double mutant, HSA32, HSP22, and HSP21 had further reduced transcript levels starting from $28 \mathrm{~h}$ compared to either single mutant (Fig. 4). This is in line with the further reduced HS memory in the double mutant and suggests that both proteins act in HS memory and cannot replace each other. In addition, we observed similar changes at the level of unspliced transcripts (Supplementary Fig. 5b), which are often used as a proxy for transcriptional activity $23,24,40$, indicating that the observed changes in transcript levels reflect changes in (ongoing) gene transcription. The expression of HS-induced nonmemory genes HSP101 and HSP70 was unaltered in all of the mutants (Fig. 4).

Consistent with previous findings ${ }^{33}, H S F A 2$ was required for type II memory at APX2, HSFA1E, MIPS2, LACS9, LPAT5, TPR1, MYB86, and DGS1 (Fig. 5). In contrast, hsfa3-1 mutants showed wild type-like enhanced re-induction of these genes. Expression of HSP101, which does not show type II transcriptional memory, was unaffected in all mutant backgrounds (Fig. 5). Thus, while HSFA2 is required for both types of transcriptional memory, HSFA3 appears specifically required for the sustained induction of HS memory-related genes (type I).

To globally assess the effects of the mutants on heat-induced gene expression during the memory phase, we performed RNAseq on Col, $h s f a 2, h s f a 3$, and double mutant seedlings that were 

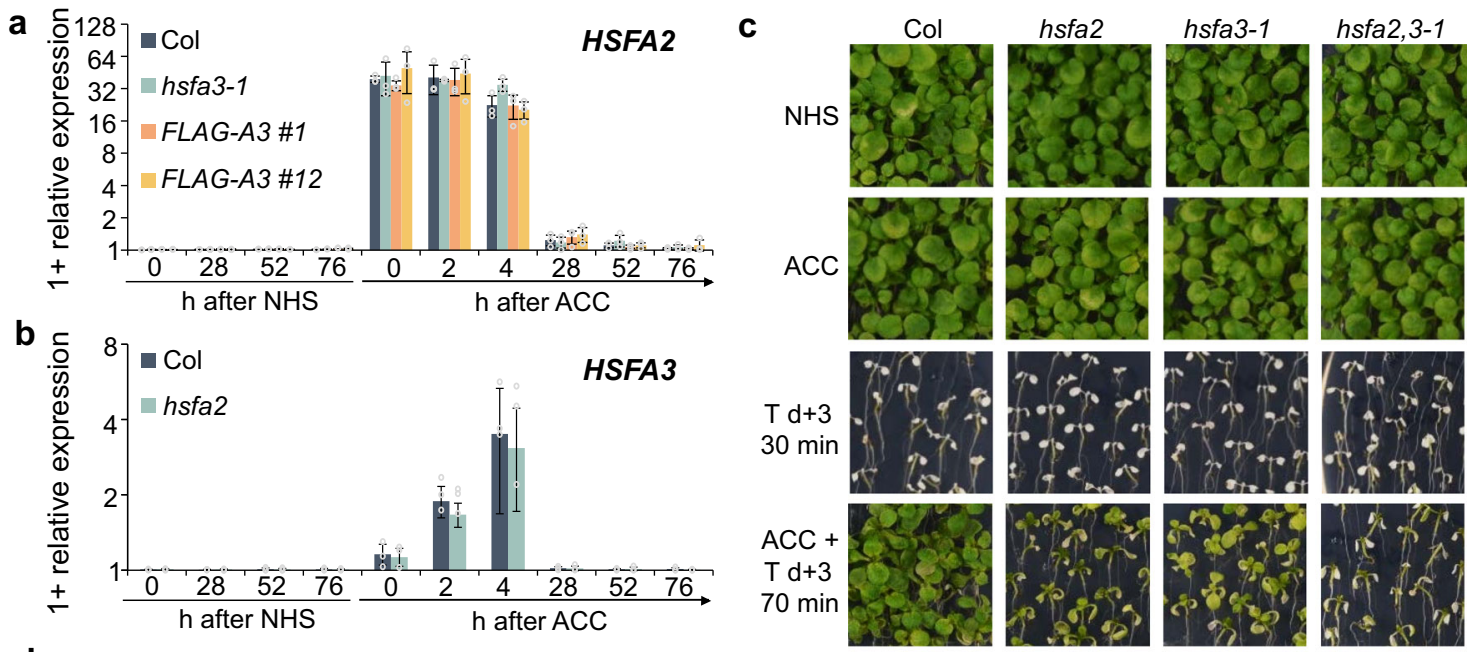

d
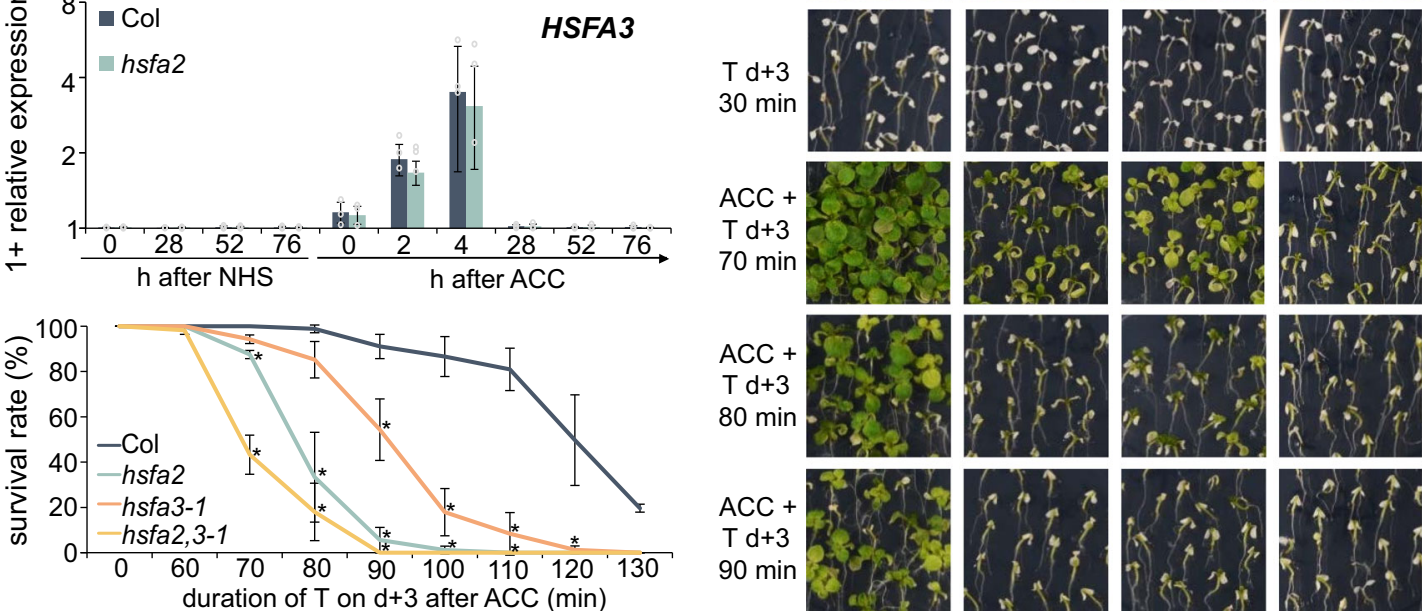

Fig. 3 HSFA2 and HSFA3 are independently induced by HS and interact genetically. a Relative transcript levels of HSFA2 in Col, hsfa3-1 and pHSFA3:: FLAG-HSFA3 lines in hsfa3-1 background as measured by qRT-PCR. b Relative transcript levels of HSFA3 in Col and hsfa2 as measured by qRT-PCR. Data are mean \pm SD of three independent experiments (a, b). c, d HS memory assay for hsfa2, hsfa3-1 and hsfa2 hsfa3-1 double mutants. 4 d-old seedlings were exposed to ACC treatment and $3 \mathrm{~d}$ later to a triggering $\mathrm{HS}$ at $44{ }^{\circ} \mathrm{C}$ for $70-130$ min. NHS, no-HS control; ACC, plants primed with an ACC treatment. Representative images (c) and survival rates (d) were recorded $14 \mathrm{~d}$ after ACC. Error bars indicate SD of three independent experiments. Asterisks mark significant differences to Col ( $p<0.05$, unpaired two-sided t-test).

subjected to an ACC treatment and recovered for $4 \mathrm{~h}, 28 \mathrm{~h}$, or 52 $\mathrm{h}$ or control samples without ACC. In Col, we identified 156 genes that showed differential gene induction $\left(\log _{2} \mathrm{FC}>1\right.$ and $p$ $<0.05$ ) at all three timepoints ("1-1-1 up"), and that we therefore call HS memory genes (Supplementary Data 1). In contrast, 3225 genes were induced specifically at $4 \mathrm{~h}$ after ACC ("1-0-0 up"). Among the memory genes, $18.6 \% / 13.5 \% / 23.7 \%$ were not induced in hsfa2/ hsfa3/ hsfa2 hsfa3 at $4 \mathrm{~h}$ after ACC (Fig. 6a, Supplementary Data 1$)$. These numbers progressively increased to $37.2 \% / 30.8 \% / 53.2 \%$ at $28 \mathrm{~h}$ and $62.2 \% / 55.8 \% / 74.4 \%$ at $52 \mathrm{~h}$ after ACC. Thus, HSFA2 and HSFA3 are partially redundantly required for sustained induction of HS memory genes and their effect becomes more pronounced as the recovery phase progresses. In contrast, of the 3225 early HS genes (1-0-0 up), $22.3 \% / 21.4 \% / 24.2 \%$ were not induced at $4 \mathrm{~h}$ in hsfa2/ hsfa3/ hsfa2 $h s f a 3$. For both groups of genes and at all three timepoints there was a large overlap between the genes with loss of upregulation in the mutants, confirming that their functions are largely overlapping (Fig. 6b). For the memory genes only, the number of genes with loss of overexpression was increased in the double mutant, confirming the cooperative effect of HSFA2 and HSFA3. The overall similar but stronger effect of the double mutant was also apparent from looking at the effect size of individual genes (Fig. 6c). Among the HS memory genes, all differentially expressed genes showed reduced induction, with the exception of two genes. In contrast, among the early HS genes, enhanced induction was more prevalent (Fig. 6c). In summary, global analysis confirmed that HSFA2 and HSFA3 function as transcriptional activators on an overlapping set of HS memory genes, where they are required for sustained induction of gene expression (type I memory).
HSFA3 and HSFA2 proteins interact. HSF proteins form multimeric complexes ${ }^{34,41}$. With the plethora of HSF proteins in $A$. thaliana, there is the potential for multiple combinations; however, which of these occur in vivo remains unresolved. We hypothesized that HSFA3 and HSFA2 may directly interact. We confirmed the interaction of HSFA 2 and HSFA 3 in the yeast-twohybrid system (Fig. 7a). The C-terminus of HSFA2 and HSFA3, which we truncated in the $\mathrm{BD}$ constructs to prevent auto activation, was dispensable for the interaction. This is consistent with the notion that the interaction is mediated by the oligomerization domain (OD, Fig. 1d). We next confirmed the interaction by in planta co-immunoprecipitation from stable transgenic lines using HSFA2-YFP 32 and FLAG-HSFA3, both expressed from their own promoters in the $h s f a 2$ hsfa3-1 double mutant background. Both proteins were strongly induced after ACC with a peak around $4 \mathrm{~h}$ into the recovery phase and they were still detectable at $76 \mathrm{~h}$ after ACC (Fig. 7b, c). FLAG-HSFA3 precipitated HSFA2-YFP at all time points where both proteins were detectable (Fig. 7b). Conversely, HSFA2-YFP was able to pull down HSFA3 (Fig. 7c). In summary, HSFA2 and HSFA3 form heteromers in planta that persist for several days after HS/ACC.

Interaction with other HSFs. Our genetic analysis indicated that in the absence of the respective other "memory" HSF, the remaining HSF still retains some activity. Thus, both HSFA2 and HSFA3 may have other binding partners. To investigate this we purified FLAG-HSFA3 and interacting proteins under no-HS conditions, $4 \mathrm{~h}$ after $\mathrm{HS}\left(1 \mathrm{~h}\right.$ at $\left.37^{\circ} \mathrm{C}\right)$, or $4 \mathrm{~h}$ after ACC from the complementing pHSFA3::FLAG-HSFA3 line and subjected them to mass spectrometry analysis (Co-IP/MS). The most frequent interacting protein as estimated by unique peptide numbers in all 

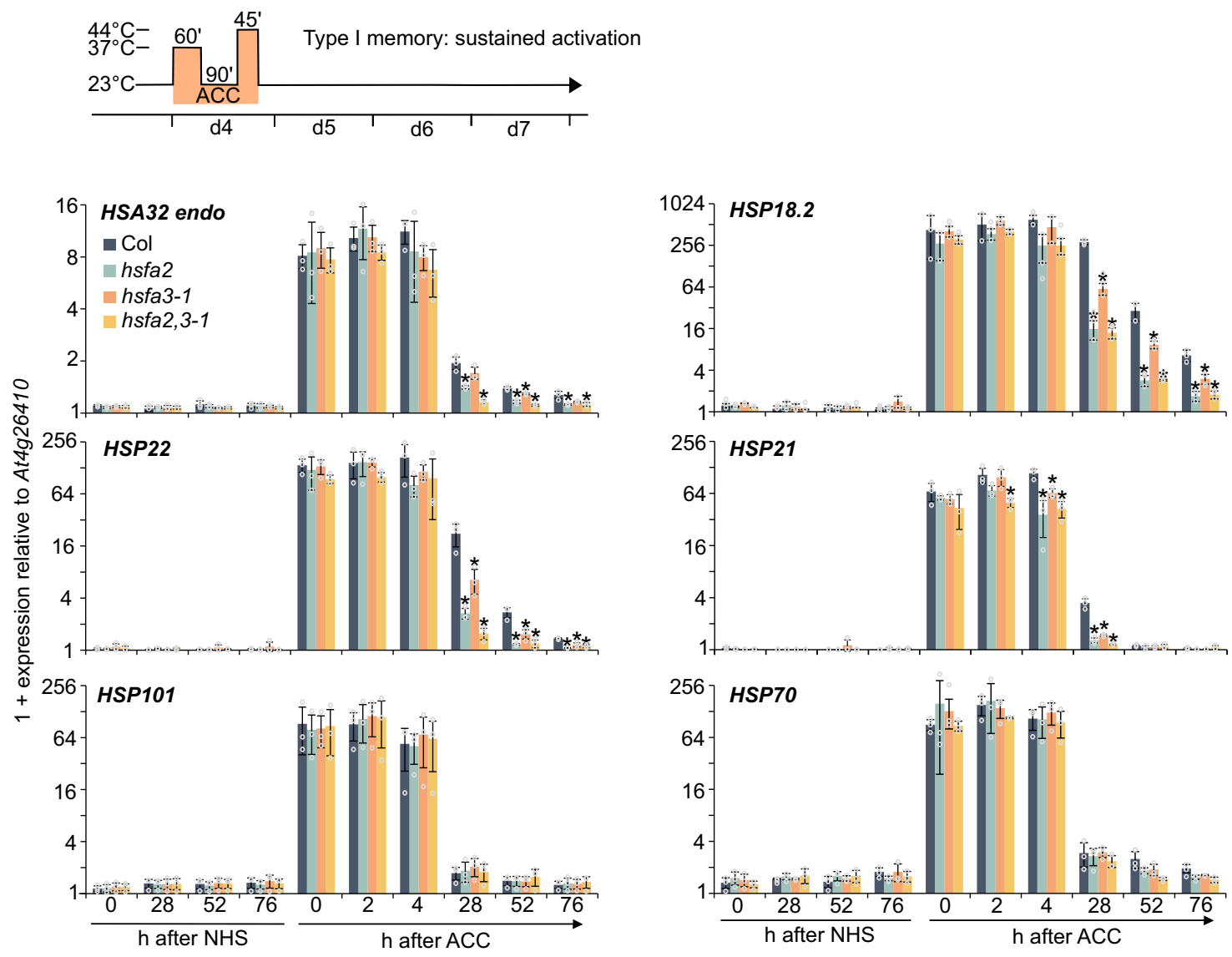

Fig. 4 Sustained induction of HS memory genes depends on HSFA2 and HSFA3. Type I transcriptional memory (sustained induction): Memory gene expression is induced by a priming ACC treatment and expression is sustained above baseline for several days. Plants were exposed to an ACC treatment and samples taken at the indicated time points during the following $76 \mathrm{~h}$. Relative transcript levels of 4 memory genes (HSA32, HSP18.2, HSP22, HSP21) and 2 HS-inducible non-memory genes (HSP101 and HSP70) were measured by qRT-PCR. Time points depict hours after end of ACC. Data are mean \pm SD of three independent experiments. Asterisks mark significant differences to Col ( $p<0.01$, unpaired two-sided t-test).

heat-treated samples was HSFA2 (Fig. 7d, Supplementary Table 2), indicating that HSFA2 is the preferred binding partner of HSFA3. In addition, we also identified HSFA1D, HSFA1B, HSFA6B, HSFA7A, and HSFA1A as interacting proteins (Fig. 7d, Supplementary Table 2). Thus, HSFA3 forms multimeric complexes with other HSF proteins, providing a tentative explanation for the residual activity of HSFA3 in the absence of HSFA2. Interestingly, we also detected interactions of HSFA3 with HSFA1 isoforms before applying any heat treatment, suggesting that the formation of HSFA1-HSFA3 complexes does not depend on a HS stimulus.

Correspondingly, we isolated HSFA2-YFP protein complexes for mass spectrometry (Fig. 7e). Since HSFA2 expression is induced faster than HSFA3 (Fig. 3a, b), samples were taken 45 min and $3 \mathrm{~h}$ after $1 \mathrm{~h} \mathrm{HS}$ at $37^{\circ} \mathrm{C}$ treatments. HSFA3 was detected with very low peptide numbers under no-HS conditions but increased at $45 \mathrm{~min}$ and $3 \mathrm{~h}$ after HS in line with the induction of HSFA3; at $3 \mathrm{~h}$ after HS HSFA3 was the second most frequent interacting protein after HSFA7A. At all time points other HSFs were recovered as HSFA2 interacting proteins. They were HSFA7A, HSFA1B, HSFA1D, HSFA1A, HSFA6B, and HSFA7B (Fig. 7e, Supplementary Table 2). With the exception of HSFA7B, we identified all HSFA2-interacting HSFs also as interactors of HSFA3, suggesting that both proteins share a common set of interactors after HS. Using in vitro pulldowns we confirmed that each HSFA2 and HSFA3 interact directly with HSFA1A, B, D, and HSFA7A (Fig. 7f).
We next asked which proteins (if any) HSFA2 and HSFA3 bind to in the absence of the respective other memory HSF. To this end, we repeated the Co-IP/MS analysis of HSFA2 or HSFA3 in the respective other mutant background in the absence of HS or $4 \mathrm{~h}$ after ACC treatment. Besides the memory HSFs, the previously identified additional interacting HSFA1s and HSFA7B were rediscovered (Supplementary Table 3). In the hsfa 2 mutant HSFA3 complexes also contained HSFA1D, HSFA1B and HSFA1A. Conversely, in the hsfa3 mutant HSFA2 complexes contained HSFA1D, HSFA1B, HSFA1A and HSFA7B. This is consistent with the idea that either memory HSF still forms complexes with additional HSF proteins in the absence of the other memory protein. However, our mutant analysis clearly indicates that these alternative complexes are less efficient in promoting HS memory.

HSFA3 and HSFA2 bind with overlapping kinetics to the same target genes. To test whether HSFA3 sustains the expression of HS memory-related genes directly, we performed time-course chromatin immunoprecipitation (ChIP) with sampling from the end of ACC until $28 \mathrm{~h}$ into the recovery phase. Indeed, we detected HS-dependent enrichment of FLAG-HSFA3 in the promoters of HSP22, HSP18.2, HSA32, and APX2 at HSEcontaining sequences (Fig. 8a, Supplementary Fig. 6a). The binding of HSFA 3 peaked $4 \mathrm{~h}$ into the recovery phase, and was still detected at $28 \mathrm{~h}$. This is consistent with HSFA3 promoting transcription for at least $24 \mathrm{~h}$ after the end of ACC. We 
Type II memory: enhanced re-induction
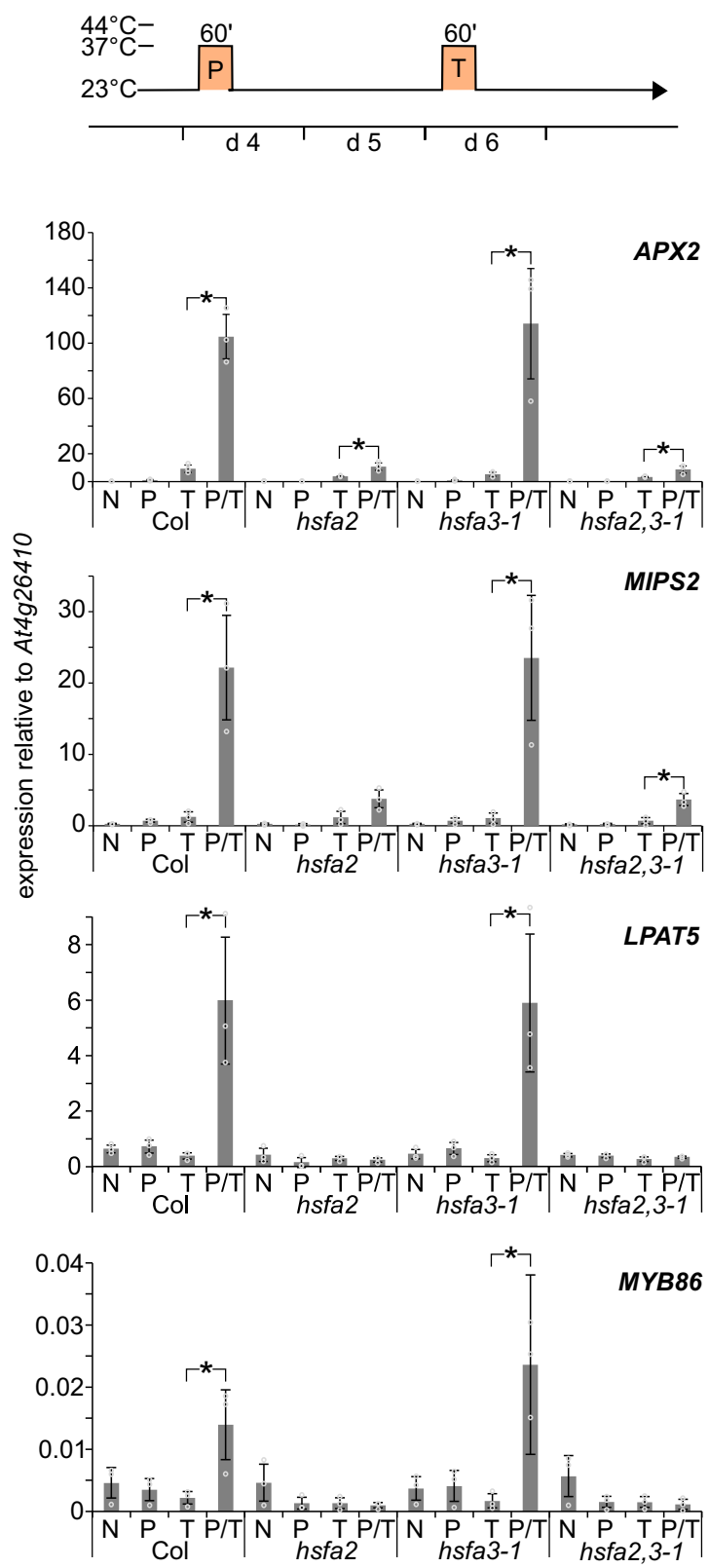
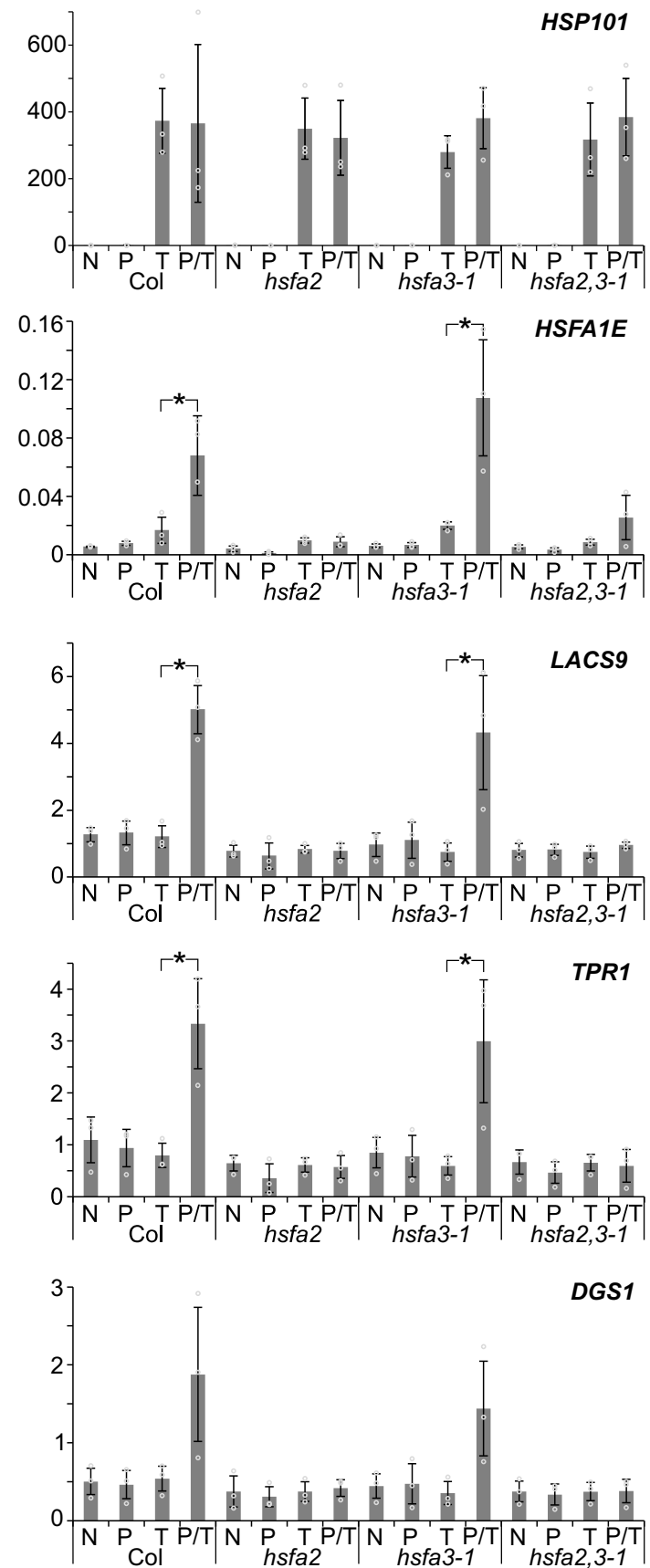

Fig. 5 Enhanced re-induction of HS memory genes depends on HSFA2 but not HSFA3. Type II transcriptional memory (enhanced re-induction): Memory gene expression is activated by a priming treatment ( $P$ ), and more highly activated by a second triggering treatment ( $T$ ) $2 \mathrm{~d}$ later. $\mathrm{P}$ and $\mathrm{T}$ treatments consist of $37^{\circ} \mathrm{C}$ for $1 \mathrm{~h}$. Relative transcript levels in enhanced re-induction experiments of eight memory genes (APX2, HSFA1E, MIPS2, LACS9, LPAT5, TPR1, MYB86, and DGS1) and one non-memory gene (HSP101) were measured by qRT-PCR. Plants were either not treated (N), only primed on $d 4(P)$, only triggered on $d 6(T)$, or primed on $d 4$ and triggered on $d 6(P+T)$. Regardless of their treatment, all samples were harvested on $d 6$ at the end of the T treatment. Data are mean \pm SD of three independent experiments. Asterisks mark significant differences to Col ( $p<0.01$, unpaired two-sided t-test).

previously found that HSFA2 is associated with these loci early after the $\mathrm{HS}^{32}$. We confirmed this in the present study using a PHSFA2::FLAG-HSFA2 line that was grown and sampled sideby-side with the FLAG-HSFA3 line (Fig. 8a, Supplementary Fig. 6a). After ACC HSFA3 and HSFA2 were also associated with the HS-inducible non-memory gene HSP101 (Fig. 8a), where they did not have any impact on gene expression (Figs. 4 and 5). Thus, while HSFA3 and HSFA2 bind to the same loci after HS, their binding kinetics differ with HSFA3 showing a delayed peak. This suggests that both proteins bind these loci with overlapping kinetics, partially together and partially using alternative HSFs as binding partners.

HSFA3 binds target loci independently of HSFA2. Given the interaction between HSFA2 and HSFA3 we wondered whether binding of HSFA3 to the promoters of target genes depends on HSFA2. We thus performed time-course ChIP with FLAGHSFA 3 in the hsfa 2 background. Overall, the HSFA3 binding dynamics to HSP22, HSP18.2, HSA32, APX2, and HSP101 were 
a Col 1-1-1 up (156)

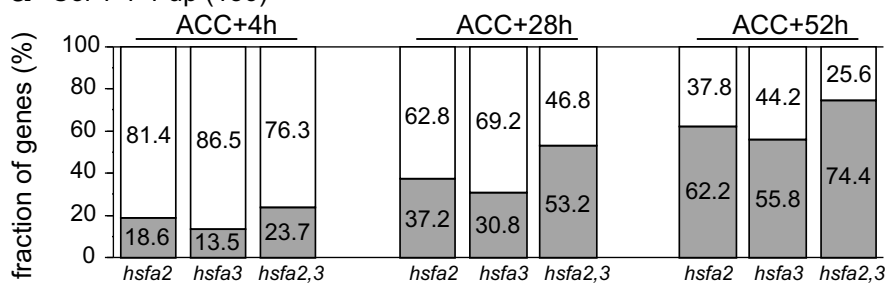

b Col 1-1-1 up
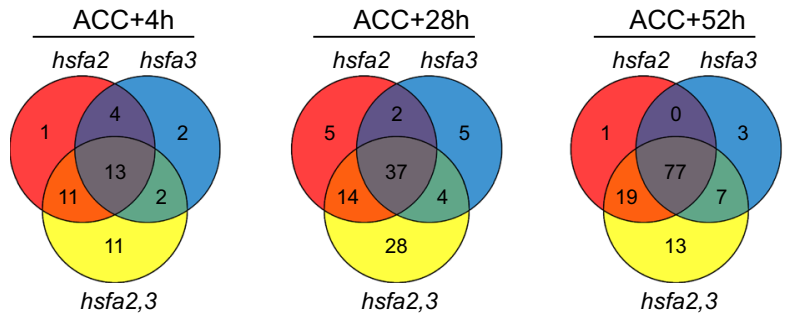

C Col 1-1-1 up (156)

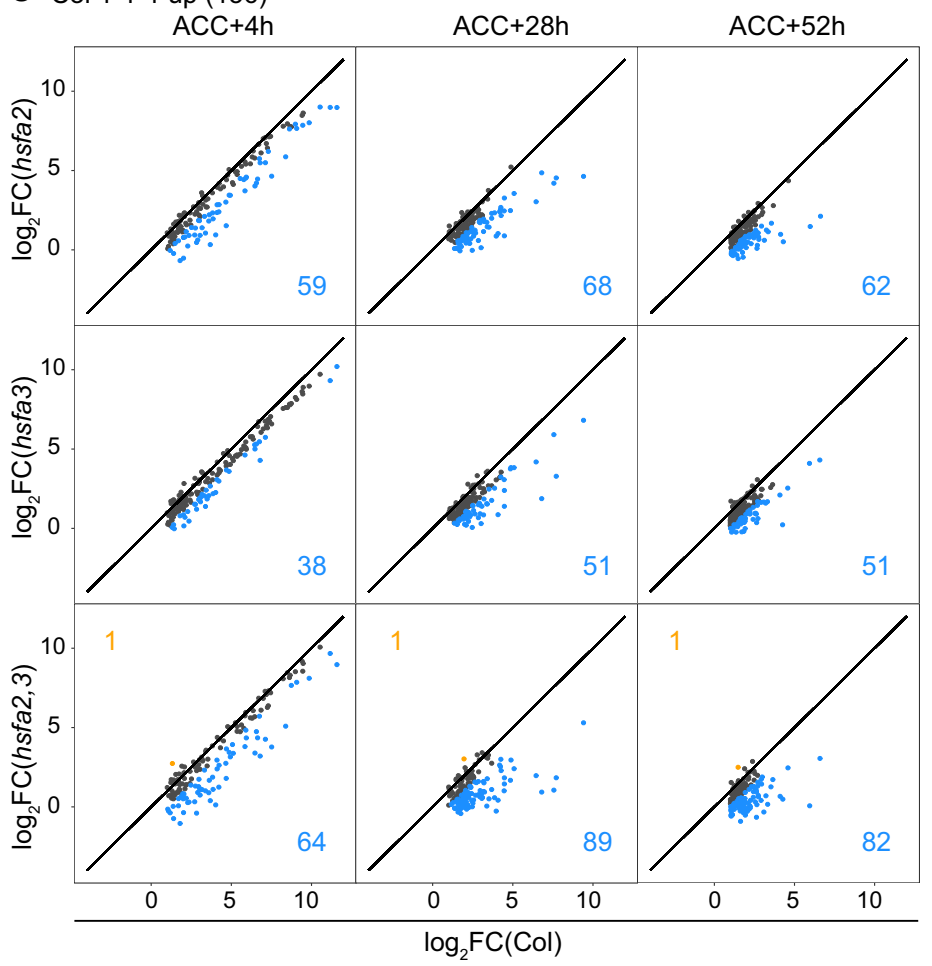

Col 1-0-0 up (3225)

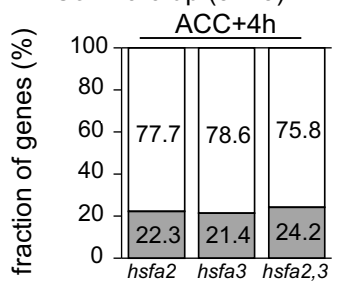

in mutant

$\square$ upregulated

not upregulated
Col 1-0-0 up

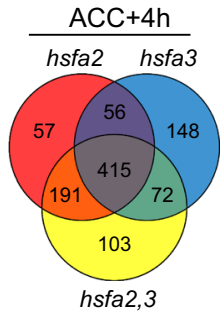

Col 1-0-0 up (3225)

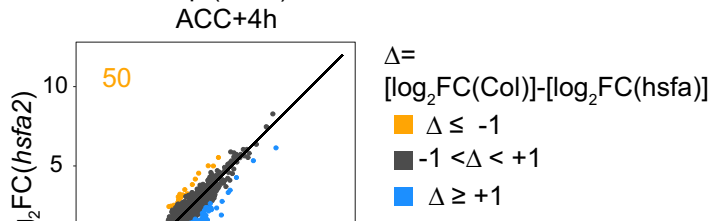

Fig. 6 HSFA2 and HSFA3 jointly promote type I transcriptional memory in a genome-wide manner. Genome-wide transcriptome profiling by RNA-seq analysis identifies $156 \mathrm{HS}$ memory genes with sustained induction above no-HS levels $\left(\log _{2} \mathrm{FC}>1, \mathrm{p}<0.05\right)$ at $4 \mathrm{~h}(\mathrm{ACC}+4 \mathrm{~h}), 28 \mathrm{~h}(\mathrm{ACC}+28 \mathrm{~h})$, and $52 \mathrm{~h}(\mathrm{ACC}+52 \mathrm{~h})$ after end of ACC treatment in Col (1-1-1 up) and 3225 genes that are induced above no-HS levels only $4 \mathrm{~h}$ after end of $\mathrm{ACC}$ treatment in Col (1-0-0 up). a Fraction of 1-1-1 up (Col) and of 1-0-0 up (Col) genes that are no longer upregulated in hsfa2, hsfa3-1 (hsfa3), and hsfa2 hsfa3-1 (hsfa23) mutants relative to their no-HS expression ( $\log _{2} \mathrm{FC} \leq 1 \mathrm{OR} \log _{2} \mathrm{FC}>1$ AND $p>0.05$, blue; $\log _{2} \mathrm{FC}>1$ AND $p>0.05$, gray). $\mathbf{b}$ Overlap of genes with loss of upregulation relative to no-HS expression ( $\log _{2} \mathrm{FC} \leq 1 \mathrm{OR} \log _{2} \mathrm{FC}>1$ AND p > 0.05) in hsfa2, hsfa3-1 (hsfa3), or hsfa2 hsfa3-1 (hsfa23) mutants among 1-1-1 up (Col) and 1-0-0 up (Col) genes. c Pairwise comparison of $\log _{2}$ FCs relative to no-HS expression between Col and hsfa2, hsfa3-1 (hsfa3) or hsfa2 hsfa3-1 (hsfa23) mutants of 1-1-1 up ( $\mathrm{Col}$ ) and 1-0-0 up ( $\mathrm{Col}$ ) genes. Genes in orange are more strongly induced in the mutant, genes in blue are less induced in the mutant. Colored numbers indicate the number of genes in the respective group.

very similar to those in the wild type background (Fig. 8b, Supplementary Fig. 6b). This suggests that HSFA2 is not required to recruit HSFA3 to its target loci. Moreover, these findings indicate that the loss of HS memory in $h s f a 2$ is not due to concomitant loss of HSFA3 for transcriptional activation; rather, it reinforces the idea that alternative HSF complexes have differential capacity to activate HS memory.

HSFA3 and HSFA2 jointly recruit histone H3K4 methylation. We previously found that HSFA2 is required for sustained 

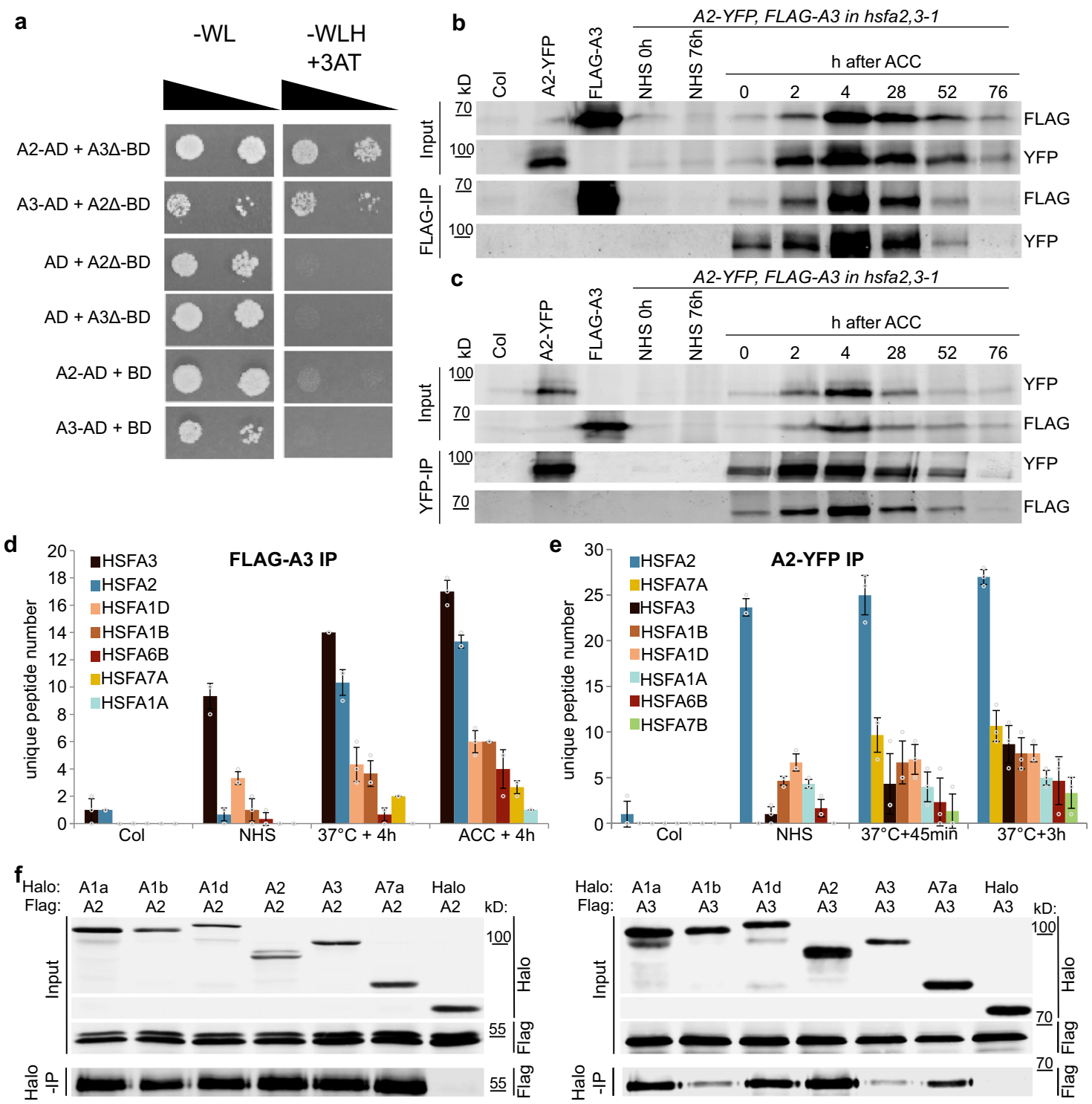

Fig. 7 HSFA2 and HSFA3 form protein complexes during HS memory. a HSFA2 and HSFA3 interact in the yeast-two-hybrid assay. The C-terminal transactivating domains of HSFA2 and HSFA3 were deleted (A2 $\Delta$ aa 1-269, A3s aa 1-275) when fused to the GAL4-DNA-binding domain (BD) to prevent auto activation. Serial dilutions were grown on -WL medium (not selecting for interaction) or on -WLH medium supplemented with 50 mM 3-AT to check for protein-protein interactions. b, c HSFA2 and HSFA3 interact in planta as shown by co-immunoprecipitation experiments: transgenic lines carrying both pHSFA2::HSFA2-YFP and pHSFA3::FLAG-HSFA3 constructs in the hsfa2 hsfa3-1 double mutant background were subjected to an ACC treatment and samples were taken at the indicated time points. Immunoprecipitation of FLAG-HSFA3 yielded the HSFA2-YFP protein (b) and immunoprecipitation of HSFA2-YFP yielded the FLAG-HSFA3 protein (c) at all time points examined. No bands of similar size were co-purified in non-treated plants (NHS $0 \mathrm{~h}$ and NHS $76 \mathrm{~h}$ ), single transgenic lines or $\mathrm{Col}$ plants sampled at $4 \mathrm{~h}$ after ACC. A representative experiment from 3 independent experiments is shown. $\mathbf{d}$, e Interacting HSF proteins as identified by co-immunoprecipitation of FLAG-HSFA3 (d) or HSFA2-YFP (e) followed by mass spectrometry after the indicated treatments $\left(37^{\circ} \mathrm{C}\right.$ treatment was for $1 \mathrm{~h}$ ). Average numbers of unique peptides are given for all HSF proteins identified (cf. Supplementary Table 2). Data are mean \pm SD of three independent experiments. Note that the HSF proteins are sorted according to the number of peptides recovered and this differs for HSFA2- and HSFA3-co-purified proteins, the same color code is used in (d) and (e). $\mathbf{f}$ In vitro pulldown of HSFA proteins. Pairs of the indicated Halo-tagged and FLAGtagged HSF proteins were co-translated in vitro and purified with anti-Halo beads. The Halo-tag alone was used as a negative control. A representative experiment from 3 independent experiments is shown.

enrichment of $\mathrm{H} 3 \mathrm{~K} 4$ trimethylation (H3K4me3) at memoryrelated genes after $\mathrm{HS}^{32}$. In hsfa2 mutants, H3K4me3 enrichment was strongly reduced but not completely abolished. To test the role of HSFA3 in sustained H3K4me3 enrichment, we analyzed $\mathrm{H} 3 \mathrm{~K} 4 \mathrm{me} 3$ levels in the double mutant and either single mutant at $28 \mathrm{~h}$ and $52 \mathrm{~h}$ after ACC. Indeed, in either single mutant $\mathrm{H} 3 \mathrm{~K} 4 \mathrm{me} 3$ enrichment after ACC was reduced to an intermediate level at HSP22; at APX2, HSFA3 was dispensable for $\mathrm{H} 3 \mathrm{~K} 4 \mathrm{me} 3$ enrichment, however, at both loci $\mathrm{H} 3 \mathrm{~K} 4 \mathrm{me} 3$ was more strongly reduced in the hsfa2 hsfa3-1 double mutant (Fig. 9). In contrast, H3K4me3 enrichment at HSP101 was not affected in either of the genotypes tested. Over all genotypes and assayed regions, histone H3 enrichment decreased after ACC (Supplementary Fig. 7), consistent with previous findings ${ }^{24}$. In summary, our findings suggest that HSFA2 and HSFA3, despite the strong phenotypes of the single mutants, show functional redundancy at the level of 
a
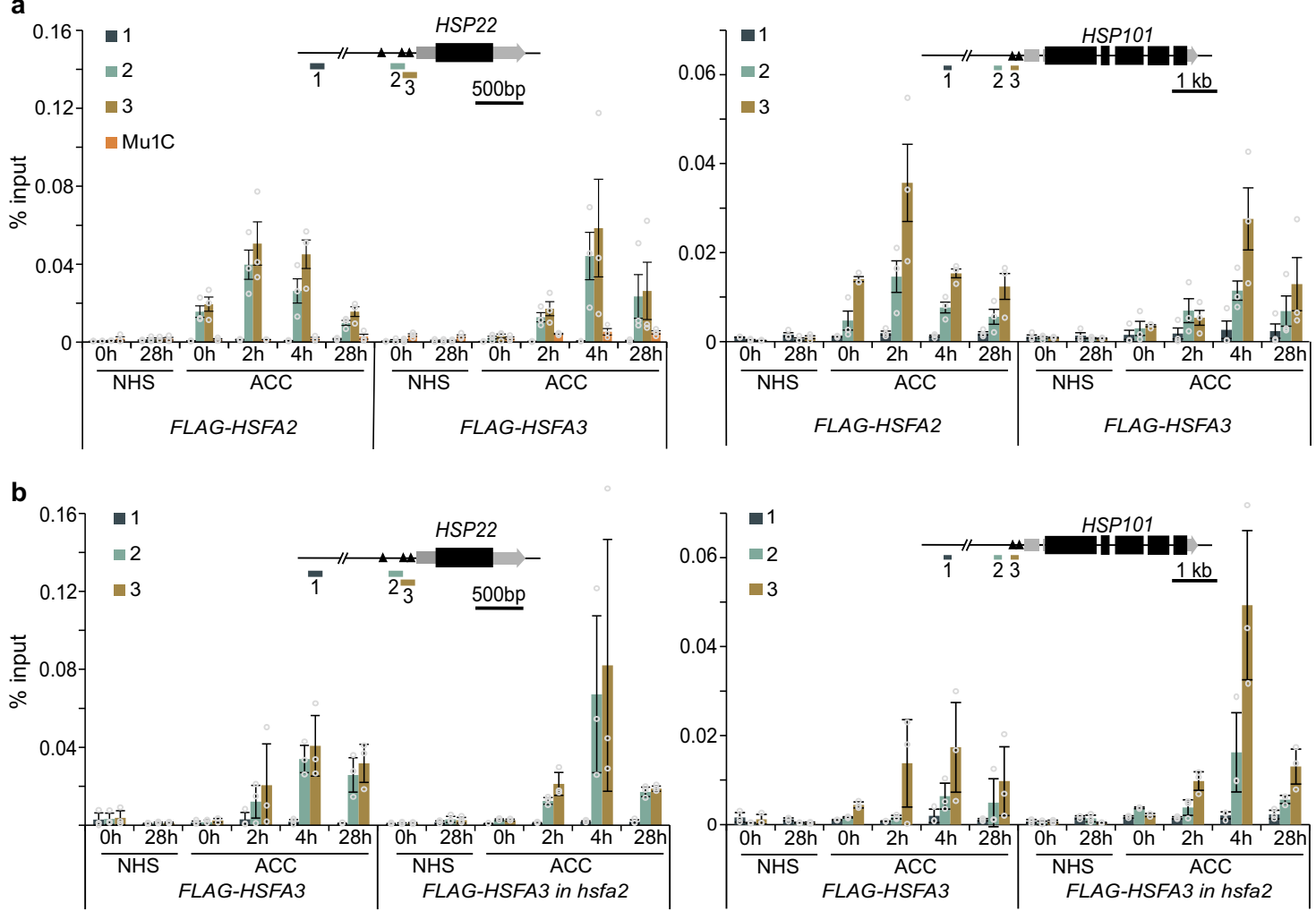

Fig. 8 HSFA2 and HSFA3 bind to memory gene promoters directly and independently. a Occupancy of HSFA2 and HSFA3 as determined by ChIP-qPCR from pHSFA2::FLAG-HSFA2 and pHSFA3::FLAG-HSFA3. b Occupancy of HSFA3 as determined by ChIP-qPCR from pHSFA3::FLAG-HSFA3 in the wild type or hsfa2 mutant background. Enrichment normalized to Input (Data are mean \pm SD) from three independent experiments is shown for the HS memory gene HSP22 and the non-memory gene HSP101. The transposon Mu1c is shown as a non HS-responsive locus. Time points are given in h after end of ACC treatment. For each locus one control amplicon situated approximately $3 \mathrm{~kb}$ upstream is shown alongside the amplicon covering heat shock elements (black triangles) in the promoter (inset gene models).

physiological HS memory, sustained memory-related gene activation, and hyper-methylation of $\mathrm{H} 3 \mathrm{~K} 4 \mathrm{me} 3$.

HSFA2 and HSFA3 can substitute for each other. The strong phenotypes of the single mutants may be caused by partially nonoverlapping expression domains or by sub-functionalization at the protein level. To discriminate between these possibilities, we performed complementation analyses with HSFA2 and HSFA3 proteins that were expressed under the control of the respective other promoter. We first expressed the FLAG-HSFA3 coding region from the $P H S F A 2$ promoter, which is activated earlier after HS than pHSFA3 (cf. Fig. 3a, b). This construct was able to partially complement the physiological HS memory phenotype of hsfa2 (Fig. 10a, Supplementary Fig. 8a, d), suggesting that the HSFA3 protein can (partially) take over HSFA2 function, when supplied from the HSFA2 promoter. The PHSFA2::FLAG-HSFA3 construct also partially complemented the hsfa3-1 mutant (Fig. 10a). We conversely asked whether the early induction of HSFA2 is required for HS memory by expressing the complementing HSFA2-YFP coding region under the control of PHSFA3. Indeed, $P H S F A 3$ - and $P H S F A 2$-driven HSFA2-YFP, respectively, rescued the $h s f a 2$ mutant phenotype in part (Fig. 10a). Finally, expression of HSFA2-YFP from $p H S F A 3$ was sufficient to restore HS-memory in hsfa3-1, suggesting that both proteins carry out the same function. In contrast, expressing FLAG-HSFA1D from the PHSFA3 promoter failed to complement the hsfa3-1 mutant (Supplementary Fig. 8e-g), indicating that HSFA2 and HSFA3 have a specialized protein function that is absent from HSFA1D.
HSFA2 but not HSFA3 is required for type II transcriptional memory after HS (Fig. 5). This is surprising in light of the above finding that both proteins appear to carry out the same functions. To further investigate this, we tested whether HSFA3 could substitute for HSFA2 regarding type II memory if expressed from pHSFA2. Introduction of pHSFA2::FLAG-HSFA3 into hsfa2 restored the enhanced re-induction of $A P X 2$ after recurring $\mathrm{HS}$, suggesting that HSFA3 is able to mediate type II memory when supplied under the correct promoter (Fig. 10b, c). Conversely, pHSFA3::HSFA2-YFP rescued type II transcriptional memory defects of $A P X 2$ in the $h s f a 2$ mutant. In summary, the promoter swapping experiments indicate that there is no clear qualitative difference between HSFA2 and HSFA3 protein functions. In the absence of HSFA2-HSFA3 heteromers, increased protein levels and correct timing of expression partially compensate for the missing partner.

\section{Discussion}

Here, we identified HSFA3 as an essential component of HS memory in A. thaliana. We show that HSFA3 is required for sustained induction of several HS memory-related genes through direct gene activation and recruitment of histone H3K4 hypermethylation. Previously, only HSFA2 was implicated in HS memory. We demonstrate that HSFA3 binds to HSFA2 to form heteromeric complexes that are highly effective at promoting HS memory.

HSFA2 and HSFA3 show different expression dynamics and this may allow fine-tuning of HS (memory) responses according to the actual environmental conditions. HSFA2 is a direct target 

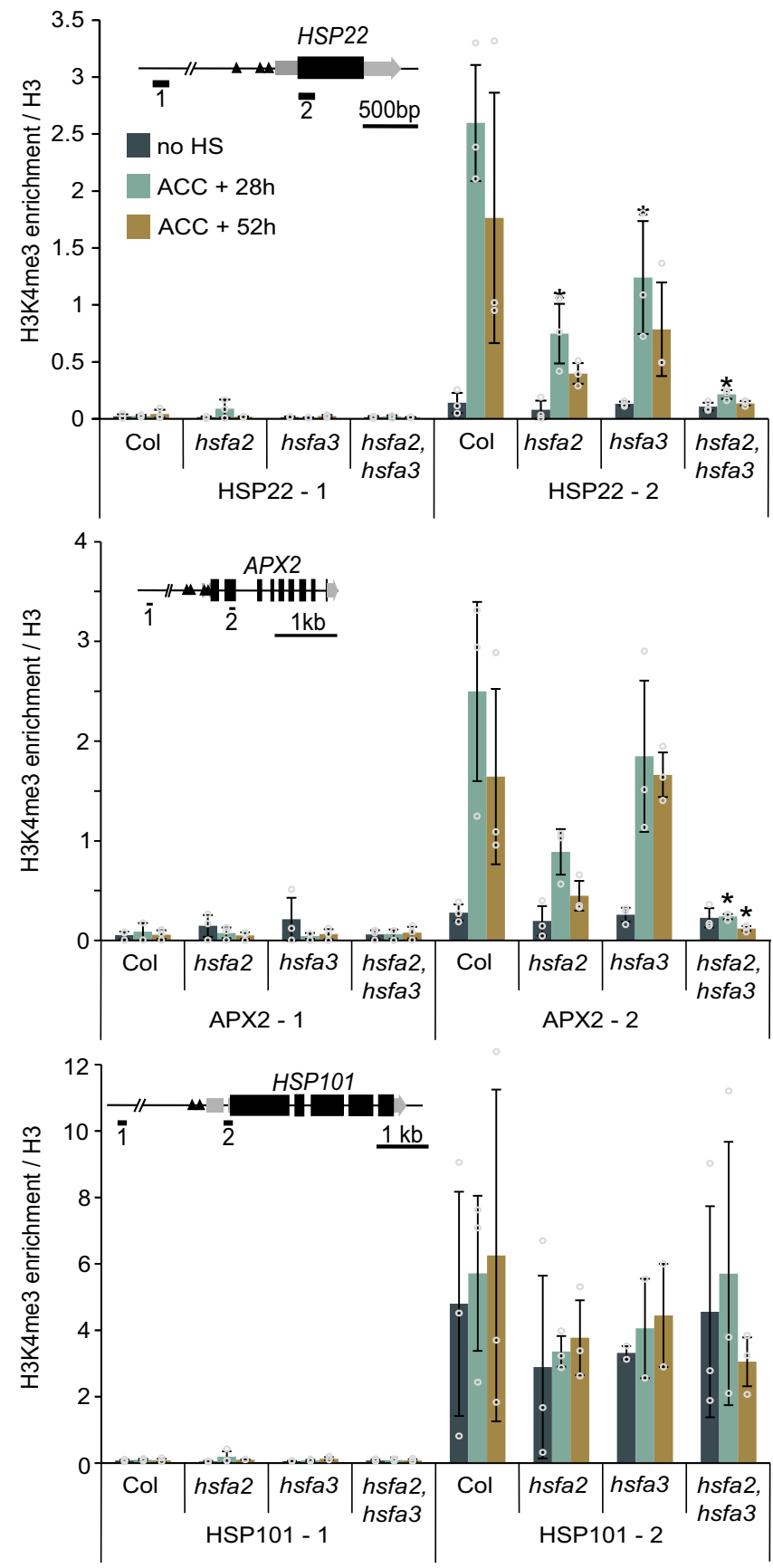

Fig. 9 H3K4me3 deposition depends on HSFA2 and HSFA3. Enrichment of H3K4me3 over H3 for the HS memory genes HSP22 and APX2, and the non-memory gene HSP101 as determined by ChIP-qPCR from three independent experiments. For each locus one control amplicon situated approximately $3 \mathrm{~kb}$ upstream is shown alongside the amplicon covering the transcriptional start site (inset gene models). Time points are given in $\mathrm{h}$ after end of $A C C$ treatment. Data are mean \pm SD. Asterisks mark significant differences relative to $\mathrm{Col}(p<0.05$, unpaired two-sided t-test).

gene of HSFA1 and induced very rapidly after the onset of $\mathrm{HS}^{22,30}$. In contrast, HSFA1s or HSFA2 do not directly induce HSFA3 (Fig. 10d). Rather, HSFA3 is activated by DREB2A, which in turn is activated by HSFA1A, and the related DREB2B and DREB2C ${ }^{30,37-39}$. DREB2A and DREB2B are also induced by drought and high salinity stress, and DREB2A is in addition regulated at the posttranslational level ${ }^{39,42,43}$. Under our conditions, HSFA 3 expression is primarily induced during the recovery phase, which is in full agreement with its function during HS memory. HSFA3 and its presumed activator DREB2B were reported as the only two transcription factors specifically enriched in acclimated plants, but not by direct exposure to acute $\mathrm{HS}^{37,44}$. Another study reported HSFA3 expression peaking after $10 \mathrm{~h}$ of continuous $37^{\circ} \mathrm{C}$ treatment ${ }^{38}$. These studies provided inconclusive evidence regarding the functional involvement of HSFA3 in HS responses. Our finding that HSFA3 has a specific role in HS memory unifies these studies and assigns a clear function to HSFA3.

To further assess the significance of the different expression dynamics, we performed promoter swapping experiments. Both HSFA2 and HSFA3 rescued the other respective mutant when expressed from the corresponding promoter at least partially. While HSFA3 was not required for enhanced re-induction after a second HS (type II memory), it was able to partially complement the type II memory defects of $h s f a 2$ when expressed from pHSFA2, suggesting that the early induction of HSFA2 contributes to type II memory. Importantly, HSFA1D was not able to rescue the $h s f a 3$ mutant, indicating functional specialization at the protein level. Thus, HSFA2 and HSFA3 appear to have similar protein properties enabling them to recruit specific transcriptional co-activators or H3K4 methyltransferases, and these appear distinct from the remainder of the HSF family. It remains an open question whether this recruitment occurs through direct proteinprotein interactions or through other proteins, e. g. components of the general transcriptional machinery. H3K4 methylation is deposited by the COMPASS complex and is required for efficient transcriptional elongation ${ }^{45-48}$. This is critical for transcriptional regulation in development and stress response in animals, where release of paused RNA Pol II into elongation is a limiting step $^{49,50}$. Understanding the molecular basis for the memoryspecific function of HSFA2 and HSFA3 will be an important goal of future work.

In yeast and animals, HSFs are present as trimers or hexamers (where two trimers combine), and a similar structure has been proposed for plant HSF complexes ${ }^{18,20,34,35,51}$. HSFA2 and HSFA3 associate with each other during the three days following a priming HS. HSFA3 binding to its target sites was independent of HSFA2, suggesting that it also forms functional complexes with other HSFs that may also be represented in the trimeric HSFA2/HSFA3/X complexes. We identified several HSFA1s (1 A, 1B, 1D) as well as HSFA7A, and HSFA6B as direct interactors of both HSFA2 and HSFA3. Indeed, we showed that binding partners of both memory HSFs in the absence of the respective other memory HSF contained the same HSFs that were found in the presence of the other memory HSF. This supports the notion that despite their overall promiscuity, only complexes that contain both HSFA2 and HSFA 3 have full capacity to activate HS memory (Fig. 10e). If HSFs assemble in heteromeric trimers with varying components, these complexes may vary in their temporal regulation, co-activator activity and target specificity; they may serve to integrate responses to different environmental cues. Noteworthy, HSFA3 responds to oxidative stress and both HSFA2 and HSFA3 are activated by excess light, while HSFA6B is activated by salt stress, osmotic stress and $\mathrm{ABA}^{52-54}$.

This work has started to unravel the in vivo complexity and dynamics of plant HSF complexes. HSFA2 and HSFA3 share a specific ability to recruit transcriptional co-activators and histone H3K4 methyltransferases during HS memory, which other HSFA proteins cannot do in their absence. HSFA2 and HSFA3 are found in heteromeric complexes together with additional HSFs, in particular HSFA1s. Maximal HS memory activation likely depends on the formation of heteromeric HSFA2/HSFA3/X trimers (Fig. 10e). The surprisingly strong single mutant phenotypes 
a
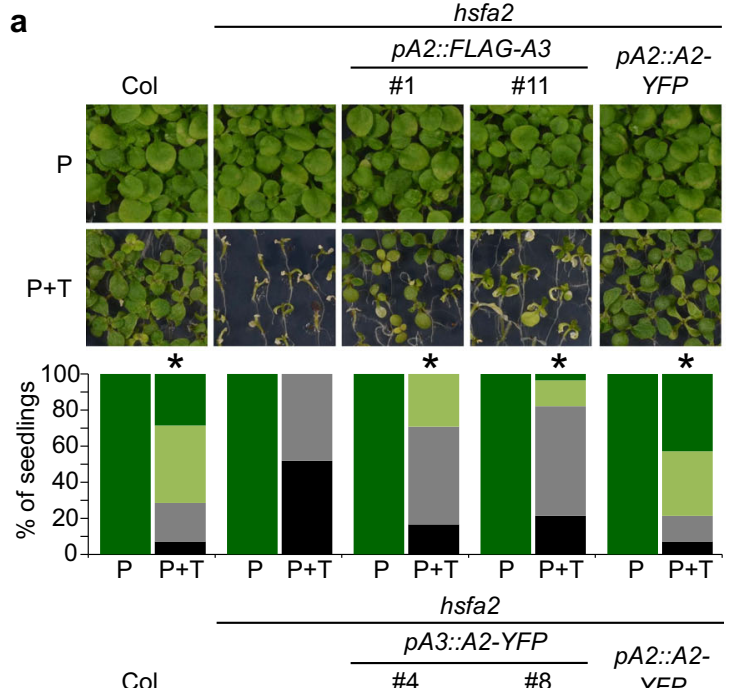

YFP
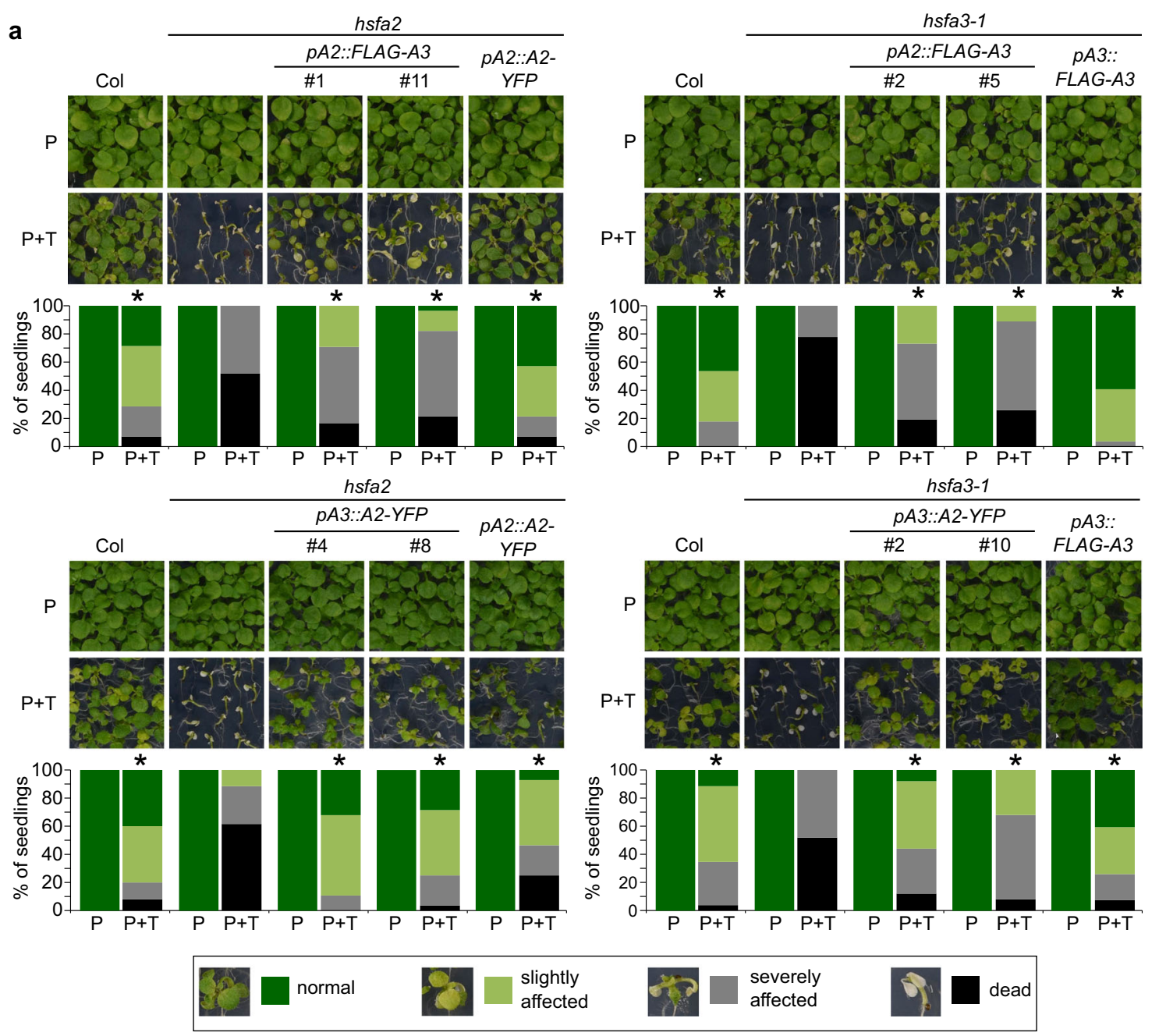

b
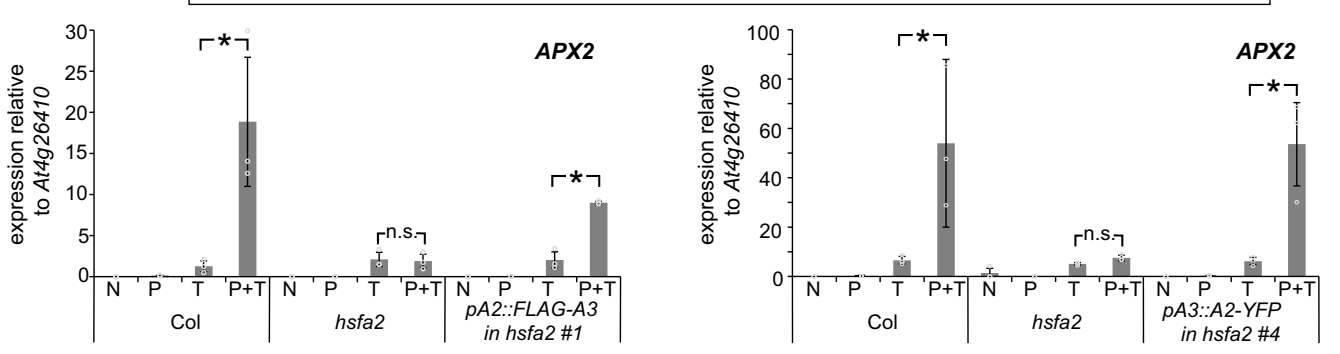

C
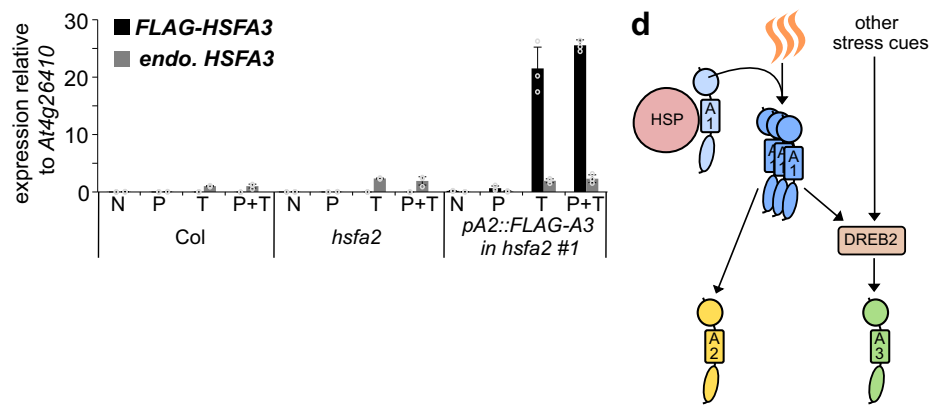

$\mathbf{e}$

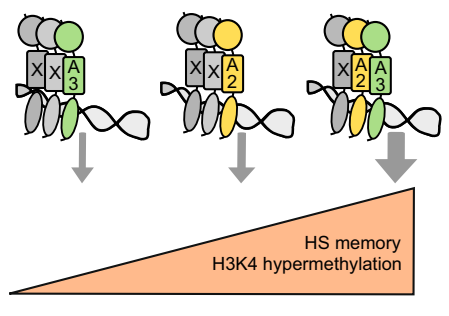

of hsfa2 and hsfa3 support this model; in the mutant backgrounds, trimers only contain a single memory HSF, resulting in much less efficient activation of transcriptional memory (Fig. 10e). The partial complementation of $h s f a 2$ single mutants by an additional copy of HSFA3 and vice versa would then be due to a higher overall abundance of such less efficient, singlememory HSF-containing complexes. In summary, our work has begun to shed light on the composition and specialized functions of in vivo HSF complexes in A. thaliana, resulting in testable predictions about a super-memory HSF complex. Ultimately, understanding the function of HSF complexes in heat shock response and transcriptional memory at a detailed biochemical level will provide targets for engineering crop plants that are more resilient to temperature extremes. 
Fig. 10 Promoter swapping indicates that HSFA2 and HSFA3 proteins carry out the same functions and working model. a $F L A G-H S F A 3$ expressed from the HSFA2 promoter complements hsfa2 and hsfa3-1 mutants in HS memory assays. HSFA2-YFP expressed from the HSFA3 promoter complements hsfa2 and hsfa3-1 mutants in HS memory assays. Asterisks mark significant differences to the mutant background $(p<0.05$, Fisher's exact test, $n \geq 24$ seedlings for each timepoint and genotype). Phenotype categories used for quantification are indicated. b, c $p H S F A 2:: F L A G-H S F A 3$ and pHSFA3::HSFA2-YFP are each sufficient to rescue the type II memory defect of $h S f a 2$. Relative transcript levels in enhanced re-induction experiments shown for the memory gene APX2 (b) and endogenous HSFA3 or FLAG-HSFA3 (c) as measured by qRT-PCR. Samples were either not treated at all (N), only primed on d 4 (P), only triggered on $d 7(T)$ or primed on $d 4$ and triggered on $d 7(P+T)$. All samples were harvested at the end of the triggering HS (T) on $d 7$. Data are mean \pm SD of three independent experiments. Asterisks mark significant differences for the indicated comparisons $(p<0.01$, unpaired two-sided t-test). d, e Working model for HS memory regulation by HSFA2 and HSFA3. d Differential regulation of HSFA2 and HSFA3 fine-tunes HS responses and integrates different environmental cues. A priming HS activates HSFA1 proteins through the release from HSPs and formation of active trimers. Active HSFA1s promote the expression of HSFA2 and DREB2 genes. DREB2 in turn promotes the expression of HSFA3; since DREB2s are also induced and activated at the posttranslational level by other stress cues, this may serve to integrate different cues into the HS memory response through HSFA3. e Different HSF complexes containing HSFA2 and/or HSFA3 form after a priming HS. They vary in their capacity to activate HS memory. The most efficient HSF complex to promote HS memory contains both HSFA2 and HSFA3, while complexes with only one of the two proteins have a reduced capacity for the recruitment of H3K4 hyper-methylation and for HS memory.

\section{Methods}

Plant material and growth conditions. All $A$. thaliana lines used in this study are in the Col-0 background. The pHSA32::HSA32-LUC ${ }^{24}$ and pHSFA2::HSFA2-YFP lines $^{32}$, the $h s f a 2-1^{22}, h s f a 3-1$ (Salk_011107) ${ }^{38}$, dreb2a-1 (379F02 GABI-KAT) ${ }^{39}$ and $h s p 101^{23}$ mutants have been described. Seedlings were grown on GM medium $\left(1 \%[\mathrm{w} / \mathrm{v}]\right.$ glucose) under a $16 \mathrm{~h} / 8 \mathrm{~h}$ light/dark cycle at $23 / 21^{\circ} \mathrm{C}^{23}$. Primer sequences for genotyping are listed in Supplementary Table 4.

HS treatments. $4 \mathrm{~d}$-old seedlings were exposed to $44^{\circ} \mathrm{C}$ for $25-45$ min to examine basal thermotolerance (bTT); to examine acquired thermotolerance (aTT) they were exposed to $37^{\circ} \mathrm{C}$ for $1 \mathrm{~h}, 23^{\circ} \mathrm{C}$ for $90 \mathrm{~min}$ and $44^{\circ} \mathrm{C}$ for $160-250 \mathrm{~min}^{23}$. For HS memory assays, $4 \mathrm{~d}$-old seedlings were primed with a two-step acclimation (ACC) protocol consisting of $37^{\circ} \mathrm{C}$ for $1 \mathrm{~h}, 23^{\circ} \mathrm{C}$ for $90 \mathrm{~min}$ and $44^{\circ} \mathrm{C}$ for 45 $\mathrm{min}^{23}$. Primed seedlings were exposed to a triggering HS at $44^{\circ} \mathrm{C}$ for $70-130 \mathrm{~min}$ on day 7 or $44^{\circ} \mathrm{C}$ for $30-80 \mathrm{~min}$ on day $8-10$.

\section{Construction of HSFA3 complementation and promoter swap constructs. To} obtain a genomic fragment containing the HSFA3 gene as well as flanking regions until the borders of the neighboring genes, PCR with primers 2410 containing an AscI site and 2418 containing a PacI site was performed and the resulting product was subcloned into pJET1.2 (Thermo Fisher). After sequencing, the genomic HSFA3 fragment was introduced using AscI and PacI into a pGreenII binary vector harboring a Norflurazone resistance (kindly provided by T. Laux). To obtain a FLAG-tagged version of HSFA3 driven by the native promoter the promoter flanked by $A s c \mathrm{I}$ and AgeI (primers 2410 and 2420) and $3 x F L A G-H S F A 3$ flanked by AgeI until the beginning of the downstream neighboring gene flanked by a PacI-site (Primers 2417 and 2418) were amplified and the resulting fragments subcloned into pJET1.2. After sequencing, the two fragments were combined in pJET1.2 via $A g e \mathrm{I}$ and $\mathrm{PacI}$ and the final fragment introduced into $p$ GreenII with Norflurazone resistance. In order to generate promoter swap constructs, either $p H S F A 2$ (primers 2624/2625), HSFA2-YFP (primers 2786/2787), or 3xFLAG-HSFA1D (primers 2810/ 2811) were amplified, subcloned into $P J E T 1.2$ and sequenced. $p H S F A 2$ replaced PHSFA3 and HSFA2-YFP or 3xFLAG-HSFA1D replaced $3 x F L A G-H S F A 3$ in the pHSFA3::3xFLAG-HSFA3 construct to obtain pHSFA2::3xFLAG-HSFA3, pHSFA3: HSFA2-YFP and PHSFA3::3xFLAG-HSFA1D. All constructs were introduced into the GV3101 strain of Agrobacterium tumefaciens and transformed using the floral dip method ${ }^{55}$. Primer sequences are listed in Supplementary Table 4.

RNA extraction and $\mathbf{q R T}-\mathbf{P C R}$. To examine sustained induction of gene expression, 4 d-old seedlings were exposed to an ACC treatment and samples were taken during the following $3 \mathrm{~d}$ as indicated. To study enhanced re-induction of memory genes, seedlings were treated for $1 \mathrm{~h}$ at $37^{\circ} \mathrm{C}$ on day four and again on day six or day seven as indicated. Samples including non-treated controls were taken at the end of the last HS. RNA was extracted from seedlings using hot-phenol RNA extraction: frozen tissue was ground to a powder and resuspended in $500 \mu \mathrm{l}$ homogenization buffer ( $100 \mathrm{mM}$ Tris $\mathrm{pH}$ 8.0, $5 \mathrm{mM}$ EDTA, $100 \mathrm{mM} \mathrm{NaCl}, 0.5 \%$ SDS), $250 \mu \mathrm{l}$ phenol and $5 \mu \mathrm{l} \mathrm{B}$-mercaptoethanol and incubated for $15 \mathrm{~min}$ at $60^{\circ} \mathrm{C}$. $250 \mu \mathrm{l}$ chloroform was added and samples were incubated for $15 \mathrm{~min}$ at $60^{\circ} \mathrm{C}$ before spinning $10 \mathrm{~min}$ at $13000 \mathrm{rpm}$. $550 \mu \mathrm{l}$ aqueous phase was transferred into a new tube containing 550 ul phenol:chloroform:isoamylalcohol (25:24:1), mixed and centrifuged as above. $500 \mu \mathrm{l}$ aqueous phase was transferred into a new tube containing $50 \mu \mathrm{l} 3 \mathrm{M}$ sodium acetate and $400 \mu \mathrm{l}$ isopropanol and precipitated at $-80^{\circ} \mathrm{C}$. After $30 \mathrm{~min}$ of centrifugation at $4{ }^{\circ} \mathrm{C}$ and $13000 \mathrm{rpm}$, pellets were dried and resuspended in $500 \mu \mathrm{l} \mathrm{H}$ O. $500 \mu \mathrm{l} 4 \mathrm{M} \mathrm{LiCl}$ was added and RNA was precipitated overnight at $4{ }^{\circ} \mathrm{C}$. RNA was pelleted, washed in $80 \% \mathrm{EtOH}$, dried, and resuspended in $40 \mu \mathrm{H}_{2} \mathrm{O}$. For quantitative RT-PCR, total RNA was treated with TURBO DNA-free (Ambion) and reverse transcribed with SuperScript III
(Invitrogen) according to manufacturers instructions. $0.1 \mu \mathrm{l}$ cDNA was used per $10 \mu \mathrm{l}$ QPCR reaction with GoTaq qPCR Master Mix (Promega) and LightCycler 480 (Roche $)^{23}$. All data were normalized to the reference gene At4g26410 ${ }^{56}$ using the comparative CT method. Primers are listed in Supplementary Table 4.

RNA-seq. For RNA-seq analysis, total RNA was extracted from Col-0, hsfa2, hsfa31 , and $h s f a 2$, 3-1 seedlings with RNeasy Plant Mini kit (Qiagen). On-column DNase digest of RNA was performed with RNase-Free DNase (Qiagen). RNA quality control, library preparation, and sequencing were performed by BGI Genomics (http://www.bgi.com) with the DNBseq platform generating $2 \times 150$ bp paired-end sequencing reads. Three biological replicates were generated and analyzed per treatment and genotype. Reads were mapped against the Arabidopsis thaliana reference genome (TAIR10) using STAR ${ }^{57}$ version 2.5.1a. Quantification at gene level was done using STAR with the quantMode GeneCounts option. Differential gene expression analysis was done using the $\mathrm{R}$ (https://www.r-project.org) package DESeq $2^{58}$. Only protein-coding genes were analyzed, transposable element genes were excluded. Col 1-1-1 up genes were defined as being induced above baseline non-heat stressed level (defined as $\log _{2}$ (fold change) $>1, p<0.05$ ) at $4 \mathrm{~h}, 28 \mathrm{~h}$, and $52 \mathrm{~h}$ after ACC treatment. 156 such genes were identified in the Col-0 background. Col 1-0-0 up genes were defined as being induced above baseline non-heat stressed level (defined as $\log _{2}$ (fold change) $>1, p<0.05$ ) at $4 \mathrm{~h}$ after ACC treatment, but not at $28 \mathrm{~h}$ or $52 \mathrm{~h}$ after ACC treatment. 3225 such genes were identified in the Col- 0 background. Genes were counted as "not upregulated in mutant" at $4 \mathrm{~h}, 28 \mathrm{~h}$, or $52 \mathrm{~h}$ after ACC treatment relative to no-HS level when either one of the following conditions was met: $\log _{2}$ (fold change $) \leq 1$ or $\log _{2}($ fold change $)>1, p>0.05$. Data visualizations were done using the $\mathrm{R}$ package ggplot2 (https://ggplot2.tidyverse.org).

Chromatin immunoprecipitation. All heat-treated samples were exposed to an ACC treatment, seedlings harvested at the indicated time points after ACC and cross-linked under vacuum in ice-cold MC buffer/ $1 \%(\mathrm{v} / \mathrm{v})$ formaldehyde ${ }^{59}$ for $2 \times 5 \mathrm{~min}$ for histone ChIP or $2 \times 10 \mathrm{~min}$ for $3 \times$ FLAG-HSFA2 or 3xFLAG-HSFA3 ChIP. Chromatin was extracted as follows; ${ }^{59}$ frozen tissue was ground up and resuspended in $25 \mathrm{ml} \mathrm{M} 1$ buffer, washed five times in $5 \mathrm{ml} \mathrm{M} 2$ buffer, and once in $5 \mathrm{ml} \mathrm{M} 3$ buffer with centrifugation for $10 \mathrm{~min}, 1000 \mathrm{~g}$ for each washing step. The resulting chromatin pellet was taken up in $1 \mathrm{ml}$ of sonication buffer (buffer recipes described in $^{59}$ ). Chromatin was sonified using a Diagenode Bioruptor ( 17 cycles/30 sec on/off) on low-intensity settings. For histone ChIP, equal amounts of chromatin from the same preparation were immunoprecipitated at $4{ }^{\circ} \mathrm{C}$ overnight using antibodies against H3 (ab1791, Abcam) or H3K4me3 (ab8580, Abcam). For 3xFLAG-HSFA2/3-ChIP, chromatin was incubated with anti-DYKDDDDK paramagnetic beads for $1 \mathrm{~h}$ at $4{ }^{\circ} \mathrm{C}$ and chromatin was recovered using a DYKDDDDK isolation kit (both Miltenyi Biotec). Immunoprecipitated DNA was quantified by qPCR (LightCycler480, Roche).

Yeast-two-hybrid analysis. HSFA2 and HSFA3 full and truncated (without AHA domains) coding regions were amplified and inserted into pGBKT7 and pGADT7 (Clontech), through either Gateway ${ }^{\circledR}$ technology (Invitrogen) or restriction enzyme (BamHI and EcoRI) digestion. Yeast cultures (MaV203 strain) were grown at $28^{\circ} \mathrm{C}$ on Yeast Peptone Dextrose (YPD) or Synthetic Dextrose (SD) media supplemented with selective Drop-out (DO) aminoacid mixtures. Double transformation with both pGBKT7 (bait) and pGADT7 (prey) constructs was performed according to standard protocols. Transformants were selected on SD medium supplemented with DO-Trp-Leu (SD-WL) and protein interaction was analyzed by growth on SD medium supplemented with DO-Trp-Leu-His (SD-WLH) and $50 \mathrm{mM} 3$ Amino-1,2,4-triazole (3-AT). 
Co-IP, immunoblotting, and mass spectrometry. Total native protein complexes were isolated from $1 \mathrm{~g}$ of seedlings in $4 \mathrm{ml}$ of Extraction buffer $(50 \mathrm{mM}$ Tris-HCl pH7.5, $150 \mathrm{mM} \mathrm{NaCl}$, 2\% Triton X-100, 1 Tablet complete mini Protease inhibitor cocktail (Roche) $/ 25 \mathrm{ml}$ ) and centrifuged 4 times at $4{ }^{\circ} \mathrm{C}$ and maximum speed for $10 \mathrm{~min} .100 \mu \mathrm{l}$ input was taken from the supernatant and $2 \mathrm{ml}$ protein extract were incubated with $50 \mu \mathrm{l}$ of $a$-DYKDDDDK paramagnetic beads for $1.5 \mathrm{~h}$ at $4{ }^{\circ} \mathrm{C}$. Protein complexes were recovered using a DYKDDDDK isolation kit (Miltenyi Biotec) and 3 washes with wash buffer (50 mM Tris- $\mathrm{HCl}$ pH7.5, $150 \mathrm{mM} \mathrm{NaCl}$ $0.1 \%$ Triton X-100, 1 Tablet complete mini Protease inhibitor cocktail (Roche) /25 $\mathrm{ml}$ ). For mass spectrometry, 3 more washes with $20 \mathrm{mM}$ Tris- $\mathrm{HCl}$ were performed. Proteins were eluted in $50 \mu \mathrm{l} 8 \mathrm{M}$ urea and used for immunoblotting ${ }^{32}$ with antiGFP (ab290, Abcam, 1:2000), anti-FLAG (M2, F1804, Sigma, 1:2500), anti H3 (ab1791, abcam, 1:5000) or anti-Tubulin (T5168, Sigma, 1:4000) antibodies. For mass spectrometry, eluates were further processed as described ${ }^{24}$. Briefly, eluates were diluted and digested with Trypsin (Fig. 7) or Trypsin/Lys-C Mix (Supplementary Table 3, Promega). Peptides were desalted, lyophilized and re-suspended in $20 \mu \mathrm{l} 3 \%(\mathrm{v} / \mathrm{v})$ acetonitrile, $0.1 \%(\mathrm{v} / \mathrm{v})$ formic acid. Measurements were performed on a Q Exactive Plus Orbitrap mass spectrometer coupled with an Easy nLC1000 HPLC (Thermo Fisher Scientific, Fig. 7) a Q Exactive HF (Thermo Fisher Scientific) mass spectrometer coupled with an Aquity M class UPLC (Waters, Supplementary Table 3)

In vitro pulldown assay. Coding sequences of HSFA proteins were inserted into the pIX-HALO expression vector by Gateway cloning. Primers are listed in Supplementary Table 4. For HSFA2 and HSFA3, the Halo tag was replaced by a $3 x$ FLAG tag to yield pIX-FLAG expression vectors. For each pulldown reaction, $500 \mathrm{ng}$ of each plasmid were mixed and transcription and translation were carried out in TNT wheat germ expression kits (Promega) according to manufacturer's instructions. Proteins were incubated overnight with Magne HaloTag beads (Promega), washed three times in PBS/ $0.1 \%$ NP-40, and eluted in SDS loading buffer. Samples were analyzed by SDS-page and immunoblotting using anti-FLAG (M2, F1804, Sigma, 1:2500) and monoclonal anti-Halo (G9211, Promega, 1:2000) antibodies.

Promoter analysis. The sequences for HSFA2 and HSFA3 promoters were analyzed using JASPAR ${ }^{60}$. The profiles for Arabidopsis HSF (MA1664.1, MA1665.1, MA1666.1, MA1667.1) and DREB2 (MA0986.1, MA1258.1) binding sites were selected for promoter analysis and analyzed with standard settings (profile score threshold $80 \%$ ).

Reporting Summary. Further information on research design is available in the Nature Research Reporting Summary linked to this article.

\section{Data availability}

RNA sequencing data have been deposited at NCBI GEO under accession number GSE162434. All raw data underlying the individual figures are provided as Supplementary Data. The plant materials generated and analyzed during the current study are available from the corresponding author upon request. Source data are provided with this paper.

Received: 22 November 2019; Accepted: 13 May 2021; Published online: 08 June 2021

\section{References}

1. Conrath, U. Molecular aspects of defence priming. Trends Plant Sci. 16, 524-531 (2011).

2. Hilker, M. et al. Priming and memory of stress responses in organisms lacking a nervous system. Biol. Rev. Camb. Philos. Soc. 91, 1118-1133 (2016).

3. Lämke, J. \& Bäurle, I. Epigenetic and chromatin-based mechanisms in environmental stress adaptation and stress memory in plants. Genome Biol. 18, 124 (2017).

4. Conrath, U., Beckers, G. J., Langenbach, C. J. \& Jaskiewicz, M. R. Priming for enhanced defense. Annu. Rev. Phytopathol. 53, 97-119 (2015).

5. Bäurle, I. Can't remember to forget you: Chromatin-based priming of somatic stress responses. Semin. Cell Dev. Biol. 133-139 (2017) https://doi.org/ 10.1016/j.semcdb.2017.09.032.

6. Rasmann, S. et al. Herbivory in the previous generation primes plants for enhanced insect resistance. Plant Physiol. 158, 854-863 (2012).

7. Slaughter, A. et al. Descendants of primed Arabidopsis plants exhibit resistance to biotic stress. Plant Physiol. 158, 835-843 (2012).

8. Wibowo, A. et al. Hyperosmotic stress memory in Arabidopsis is mediated by distinct epigenetically labile sites in the genome and is restricted in the male germline by DNA glycosylase activity. eLife 5, e13546 (2016).
9. Ding, Y., Fromm, M. \& Avramova, Z. Multiple exposures to drought 'train' transcriptional responses in Arabidopsis. Nat. Commun. 3, 740 (2012).

10. Kim, J. M. et al. Transition of chromatin status during the process of recovery from drought stress in Arabidopsis thaliana. Plant Cell Physiol. 53, 847-856 (2012).

11. Feng, X. J. et al. Light affects salt stress-induced transcriptional memory of P5CS1 in Arabidopsis. Proc. Natl Acad. Sci. USA 113, E8335-E8343 (2016).

12. Jaskiewicz, M., Conrath, U. \& Peterhänsel, C. Chromatin modification acts as a memory for systemic acquired resistance in the plant stress response. $E M B O$ Rep. 12, 50-55 (2011).

13. Singh, P. et al. Environmental history modulates arabidopsis pattern-triggered immunity in a HISTONE ACETYLTRANSFERASE1-dependent manner. Plant Cell 26, 2676-2688 (2014).

14. Mozgova, I. et al. Chromatin assembly factor CAF-1 represses priming of plant defence response genes. Nat. Plants 1, 15127 (2015).

15. Battisti, D. S. \& Naylor, R. L. Historical warnings of future food insecurity with unprecedented seasonal heat. Science 323, 240-244 (2009).

16. Lobell, D. B., Schlenker, W. \& Costa-Roberts, J. Climate trends and global crop production since 1980. Science 333, 616-620 (2011).

17. Richter, K., Haslbeck, M. \& Buchner, J. The heat shock response: life on the verge of death. Mol. Cell 40, 253-266 (2010).

18. Li, J., Labbadia, J. \& Morimoto, R. I. Rethinking HSF1 in stress, development, and organismal health. Trends cell Biol. 27, 895-905 (2017).

19. Gomez-Pastor, R., Burchfiel, E. T. \& Thiele, D. J. Regulation of heat shock transcription factors and their roles in physiology and disease. Nat. Rev. Mol. Cell Biol. 19, 4-19 (2018).

20. Ohama, N., Sato, H., Shinozaki, K. \& Yamaguchi-Shinozaki, K. Transcriptional regulatory network of plant heat stress response. Trends Plant Sci. 22, 53-65 (2017).

21. Charng, Y. Y., Liu, H. C., Liu, N. Y., Hsu, F. C. \& Ko, S. S. Arabidopsis Hsa32, a novel heat shock protein, is essential for acquired thermotolerance during long recovery after acclimation. Plant Physiol. 140, 1297-1305 (2006).

22. Charng, Y. Y. et al. A heat-inducible transcription factor, HsfA2, is required for extension of acquired thermotolerance in Arabidopsis. Plant Physiol. 143, 251-262 (2007)

23. Stief, A. et al. Arabidopsis miR156 regulates tolerance to recurring environmental stress through SPL transcription factors. Plant Cell 26, 1792-1807 (2014)

24. Brzezinka, K. et al. Arabidopsis FORGETTER1 mediates stress-induced chromatin memory through nucleosome remodeling. eLife 5, e17061 (2016).

25. Brzezinka, K., Altmann, S. \& Baurle, I. BRUSHY1/TONSOKU/MGOUN3 is required for heat stress memory. Plant Cell Environ. 42, 765-775 (2019).

26. Scharf, K. D., Berberich, T., Ebersberger, I. \& Nover, L. The plant heat stress transcription factor (Hsf) family: structure, function and evolution. Biochim. Biophys. Acta 1819, 104-119 (2012).

27. Ikeda, M., Mitsuda, N. \& Ohme-Takagi, M. Arabidopsis HsfB1 and HsfB2b act as repressors of the expression of heat-inducible Hsfs but positively regulate the acquired thermotolerance. Plant Physiol. 157, 1243-1254 (2011).

28. Nishizawa-Yokoi, A. et al. HsfAld and HsfAle involved in the transcriptional regulation of HsfA2 function as key regulators for the Hsf signaling network in response to environmental stress. Plant Cell Physiol. 52, 933-945 (2011)

29. Liu, H. C., Liao, H. T. \& Charng, Y. Y. The role of class A1 heat shock factors (HSFA1s) in response to heat and other stresses in Arabidopsis. Plant Cell Environ. 34, 738-751 (2011).

30. Yoshida, T. et al. Arabidopsis HsfAl transcription factors function as the main positive regulators in heat shock-responsive gene expression. Mol. Genet Genomics 286, 321-332 (2011).

31. Yeh, C. H., Kaplinsky, N. J., Hu, C. \& Charng, Y. Y. Some like it hot, some like it warm: phenotyping to explore thermotolerance diversity. Plant Sci.: Int. J. Exp. Plant Biol. 195, 10-23 (2012).

32. Lämke, J., Brzezinka, K., Altmann, S. \& Bäurle, I. A hit-and-run heat shock factor governs sustained histone methylation and transcriptional stress memory. EMBO J. 35, 162-175 (2016).

33. Liu, H. C. et al. Distinct heat shock factors and chromatin modifications mediate the organ-autonomous transcriptional memory of heat stress. Plant J. 95, 401-413 (2018).

34. Chan-Schaminet, K. Y., Baniwal, S. K., Bublak, D., Nover, L. \& Scharf, K. D. Specific interaction between tomato HsfA1 and HsfA2 creates heterooligomeric superactivator complexes for synergistic activation of heat stress gene expression. J. Biol. Chem. 284, 20848-20857 (2009).

35. Clos, J. et al. Molecular cloning and expression of a hexameric Drosophila heat shock factor subject to negative regulation. Cell 63, 1085-1097 (1990).

36. Majumdar, A., Nagaraj, R. \& Banerjee, U. strawberry notch encodes a conserved nuclear protein that functions downstream of Notch and regulates gene expression along the developing wing margin of Drosophila. Genes Dev. 11, 1341-1353 (1997).

37. Schramm, F. et al. A cascade of transcription factor DREB2A and heat stress transcription factor HsfA3 regulates the heat stress response of Arabidopsis. Plant J. 53, 264-274 (2008). 
38. Yoshida, T. et al. Functional analysis of an Arabidopsis heat-shock transcription factor HsfA3 in the transcriptional cascade downstream of the DREB2A stress-regulatory system. Biochem. Biophys. Res. Commun. 368, 515-521 (2008).

39. Sakuma, Y. et al. Dual function of an Arabidopsis transcription factor DREB2A in water-stress-responsive and heat-stress-responsive gene expression. Proc. Natl Acad. Sci. USA 103, 18822-18827 (2006).

40. Bäurle, I., Smith, L., Baulcombe, D. C. \& Dean, C. Widespread role for the flowering-time regulators FCA and FPA in RNA-mediated chromatin silencing. Science 318, 109-112 (2007).

41. Sandqvist, A. et al. Heterotrimerization of heat-shock factors 1 and 2 provides a transcriptional switch in response to distinct stimuli. Mol. Biol. Cell 20, 1340-1347 (2009).

42. Sakuma, Y. et al. Functional analysis of an Arabidopsis transcription factor DREB2A, involved in drought-responsive gene expression. Plant Cell 18, 1292-1309 (2006).

43. Morimoto, K. et al. BPM-CUL3 E3 ligase modulates thermotolerance by facilitating negative regulatory domain-mediated degradation of DREB2A in Arabidopsis. Proc. Natl Acad. Sci. USA 114, E8528-E8536 (2017).

44. Larkindale, J. \& Vierling, E. Core genome responses involved in acclimation to high temperature. Plant Physiol. 146, 748-761 (2008)

45. Ding, Y. et al. ATX1-generated H3K4me3 is required for efficient elongation of transcription, not initiation, at ATX1-regulated genes. PLoS Genet. 8 , e1003111 (2012).

46. Zhou, K., Kuo, W. H. W., Fillingham, J. \& Greenblatt, J. F. Control of transcriptional elongation and cotranscriptional histone modification by the yeast BUR kinase substrate Spt5. Proc. Natl Acad. Sci. USA 106, 6956-6961 (2009).

47. Lu, C. et al. Phosphorylation of SPT5 by CDKD;2 Is Required for VIP5 Recruitment and Normal Flowering in Arabidopsis thaliana. Plant Cell 29, 277-291 (2017).

48. Leng, X., Thomas, Q., Rasmussen, S. H. \& Marquardt, S. A G(enomic)P (ositioning)S(ystem) for Plant RNAPII Transcription. Trends Plant Sci. 25, 744-764 (2020).

49. Zeitlinger, J. et al. RNA polymerase stalling at developmental control genes in the Drosophila melanogaster embryo. Nat. Genet. 39, 1512-1516 (2007).

50. Jonkers, I. \& Lis, J. T. Getting up to speed with transcription elongation by RNA polymerase II. Nat. Rev. Mol. Cell Biol. 16, 167-177 (2015).

51. Liu, X. et al. Crystal structure of the hexamer of human heat shock factor binding protein 1. Proteins 75, 1-11 (2009).

52. Jung, H. S. et al. Subset of heat-shock transcription factors required for the early response of Arabidopsis to excess light. Proc. Natl Acad. Sci. USA 110, 14474-14479 (2013).

53. Nishizawa, A. et al. Arabidopsis heat shock transcription factor A2 as a key regulator in response to several types of environmental stress. Plant J. 48, 535-547 (2006).

54. Huang, Y.-C., Niu, C.-Y., Yang, C.-R. \& Jinn, T.-L. The heat stress factor HSFA6b connects ABA signaling and ABA-mediated heat responses. Plant Physiol. 172, 1182-1199 (2016).

55. Clough, S. J. \& Bent, A. F. Floral dip: a simplified method for Agrobacteriummediated transformation of Arabidopsis thaliana. Plant J. 16, 735-743 (1998).

56. Czechowski, T., Stitt, M., Altmann, T., Udvardi, M. K. \& Scheible, W. R. Genome-wide identification and testing of superior reference genes for transcript normalization in Arabidopsis. Plant Physiol. 139, 5-17 (2005).

57. Dobin, A. et al. STAR: ultrafast universal RNA-seq aligner. Bioinformatics 29, 15-21 (2013)

58. Love, M. I., Huber, W. \& Anders, S. Moderated estimation of fold change and dispersion for RNA-seq data with DESeq2. Genome Biol. 15, 550 (2014).
59. Kaufmann, K. et al. Chromatin immunoprecipitation (ChIP) of plant transcription factors followed by sequencing (ChIP-SEQ) or hybridization to whole genome arrays (ChIP-CHIP). Nat. Protoc. 5, 457-472 (2010).

60. Fornes, O. et al. JASPAR 2020: update of the open-access database of transcription factor binding profiles. Nucleic Acids Res. 48, D87-D92 (2020).

\section{Acknowledgements}

We thank the European Arabidopsis stock center for seeds. We thank L. Jiang, I. Koeppel, K. Sander for technical assistance and M. Lenhard for helpful comments. We thank D. Mäker and C. Schmidt for excellent plant care. IB acknowledges funding from a SofjaKovalevskaja-Award (Alexander-von-Humboldt-Foundation), CRC973 (project A2, Deutsche Forschungsgemeinschaft), and the European Research Council (ERC CoG 725295 CHROMADAPT).

\section{Author contributions}

T.F., V.O., S.A., K.B., and I.B. conceived and designed experiments, T.F., V.O., I.T., S.A. K.B., J.L., E.S., A.S., M.G., A.G., and C.K. performed experiments and analyzed the data. T.F. and I.B. wrote the manuscript with contributions from all authors.

\section{Funding}

Open Access funding enabled and organized by Projekt DEAL.

\section{Competing interests}

The authors declare no competing interests.

\section{Additional information}

Supplementary information The online version contains supplementary material available at https://doi.org/10.1038/s41467-021-23786-6.

Correspondence and requests for materials should be addressed to I.B.

Peer review information Nature Communications thanks Palak Chaturvedi and the other, anonymous reviewer(s) for their contribution to the peer review of this work. Peer review reports are available.

Reprints and permission information is available at http://www.nature.com/reprints

Publisher's note Springer Nature remains neutral with regard to jurisdictional claims in published maps and institutional affiliations.

pen Access This article is licensed under a Creative Commons Attribution 4.0 International License, which permits use, sharing, adaptation, distribution and reproduction in any medium or format, as long as you give appropriate credit to the original author(s) and the source, provide a link to the Creative Commons license, and indicate if changes were made. The images or other third party material in this article are included in the article's Creative Commons license, unless indicated otherwise in a credit line to the material. If material is not included in the article's Creative Commons license and your intended use is not permitted by statutory regulation or exceeds the permitted use, you will need to obtain permission directly from the copyright holder. To view a copy of this license, visit http://creativecommons.org/ licenses/by/4.0/.

(C) The Author(s) 2021 